\title{
Retinoid X receptors orchestrate osteoclast differentiation and postnatal bone remodeling
}

\author{
María P. Menéndez-Gutiérrez,, ${ }^{1}$ Tamás Röszer, ${ }^{1,2}$ Lucía Fuentes, ${ }^{1}$ Vanessa Núñez, ${ }^{1}$ Amelia Escolano, ${ }^{3}$ Juan Miguel Redondo, ${ }^{3}$ \\ Nora De Clerck, ${ }^{4}$ Daniel Metzger, ${ }^{5}$ Annabel F. Valledor, ${ }^{6}$ and Mercedes Ricote ${ }^{1}$ \\ 'Department of Cardiovascular Development and Repair, Centro Nacional de Investigaciones Cardiovasculares (CNIC), Madrid, Spain. ${ }^{2}$ Institut für Allgemeine Zoologie und Endokrinologie, Universität Ulm, Ulm, \\ Germany. ${ }^{3}$ Department of Vascular Biology and Inflammation, CNIC, Madrid, Spain. ${ }^{4}$ Microtomography Research Group, University of Antwerp, Antwerp, Belgium. Institut de Cénétique et de Biologie \\ Moléculaire et Cellulaire, CNRS UMR 7104, INSERM U964, Université de Strasbourg, Illkirch, France. ${ }^{6}$ Department of Physiology and Immunology, Faculty of Biology, University of Barcelona, Barcelona, Spain.
}

Osteoclasts are bone-resorbing cells that are important for maintenance of bone remodeling and mineral homeostasis. Regulation of osteoclast differentiation and activity is important for the pathogenesis and treatment of diseases associated with bone loss. Here, we demonstrate that retinoid X receptors (RXRs) are key elements of the transcriptional program of differentiating osteoclasts. Loss of RXR function in hematopoietic cells resulted in formation of giant, nonresorbing osteoclasts and increased bone mass in male mice and protected female mice from bone loss following ovariectomy, which induces osteoporosis in WT females. The increase in bone mass associated with RXR deficiency was due to lack of expression of the RXR-dependent transcription factor v-maf musculoaponeurotic fibrosarcoma oncogene family, protein B (MAFB) in osteoclast progenitors. Evaluation of osteoclast progenitor cells revealed that RXR homodimers directly target and bind to the Mafb promoter, and this interaction is required for proper osteoclast proliferation, differentiation, and activity. Pharmacological activation of RXRs inhibited osteoclast differentiation due to the formation of RXR/liver X receptor (LXR) heterodimers, which induced expression of sterol regulatory element binding protein-1c (SREBP-1c), resulting in indirect MAFB upregulation. Our study reveals that RXR signaling mediates bone homeostasis and suggests that RXRs have potential as targets for the treatment of bone pathologies such as osteoporosis.

\section{Introduction}

Bone undergoes constant remodeling by balancing the antagonistic activities of bone-forming osteoblasts and bone lytic osteoclasts (1-3). Bone loss resulting from increased osteoclast differentiation and activity $(1,4)$ can lead to osteoporosis and its secondary complications, such as increased fracture risk and impaired bone regeneration, which are prevalent pathologies $(1,5,6)$. Current antiresorptive therapies are limited by side effects and inadequate long-term compliance (7). Identification of molecules that regulate osteoclast differentiation and activity thus can help improve the treatment of bone loss.

Retinoid X receptors (RXRs) are members of the nuclear receptor superfamily of ligand-dependent transcription factors that regulate a variety of physiological processes, including cell differentiation, lipid and glucose metabolism, and immune responses (8). There are $3 \mathrm{RXR}$ isotypes, $\mathrm{RXR} \alpha$ (NR2B1), RXR $\beta$ (NR2B2), and $\mathrm{RXR} \gamma$ (NR2B3), which all show tissue-specific expression (8). RXRs occupy a central position in the nuclear receptor superfamily because they form heterodimers with many other family members. In addition, RXRs are able to activate transcription as homodimers, generating an as-yet little-explored complexity of RXR-dependent

Authorship note: María P. Menéndez-Gutiérrez and Tamás Rőszer contributed equally to this work.

Conflict of interest: The authors have declared that no conflict of interest exists. Submitted: May 28, 2014; Accepted: December 2, 2014.

Reference information: J Clin Invest. 2015;125(2):809-823. doi:10.1172/JCI77186. gene regulation (8). Despite the importance of RXR-controlled gene transcription, RXRs have been mainly studied as subordinate partners of other nuclear receptors, and very little is known about their in vivo functions (8). Pharmacological studies have identified RXRs as important modulators of glucose metabolism $(8,9)$, making them potential targets for the treatment of insulin resistance (IR). However, whether RXR modulation might have any impact on osteoporosis, a pathology often associated with IR (10), is not known.

Here, we have uncovered a role of RXR signaling in osteoclastogenesis during physiological and pathological bone remodeling. Using hematopoietic-specific RXR-deficient mice as well as molecular and pharmacological approaches, we show that RXRs control osteoclast proliferation, differentiation, and activation through dual mechanisms that converge on the upregulation of the transcription factor $\mathrm{v}$-maf musculoaponeurotic fibrosarcoma oncogene family, protein B (MAFB). Under physiological conditions, RXR homodimers control Mafb expression in osteoclast progenitors. In contrast, pharmacological activation of RXRs triggers a second mechanism involving heterodimerization with liver $\mathrm{X}$ receptor (LXR), which induces $M a f b$ expression indirectly through upregulation of the transcription factor sterol regulatory element binding protein-1c (SREBP-1c). These results suggest that bone loss can be inhibited by modulation of RXR signaling at different steps of osteoclast differentiation. Supporting this, we have found that both RXR deletion in osteoclast progenitors and pharmacological RXR activation in differentiating osteoclasts limit bone loss in ovariectomized mice. 


\section{Results}

$R X R s$ regulate osteoclastogenesis in vivo. Osteoclasts differentiate from the myeloid lineage of hematopoietic cells, which express RXR $\alpha$ and RXR $\beta$, but not RXR $\gamma$ (8). Mice with deletion of RXR in hematopoietic cells (11) or systemic loss of RXR $\beta$ (12) display normal hematopoiesis, suggesting that the $2 \mathrm{RXR}$ isotypes may compensate for the lack of each other in hematopoietic cells. To avoid this possible compensation, we conditionally deleted both $\mathrm{RXR} \alpha$ and RXR $\beta$ in hematopoietic cells by crossing mice bearing loxP-flanked RXR $\alpha$ and $\mathrm{RXR} \beta$ sequences with Mx1-Cre transgenic mice. RXR deletion was induced by neonatal injection of polyinosinic-polycytidylic acid (pI:pC). This model resulted in loss of expression of RXR $\alpha$ and RXR $\beta$ in all hematopoietic cells, osteoclast progenitors, and mature osteoclasts (RXR-KO) (Supplemental Figure 1; supplemental material available online with this article; doi:10.1172/JCI77186DS1).

We studied the skeletal phenotype of 8- and 20-week-old male mice. The total and femur levels of bone mineral density (BMD) and the bone mineral content (BMC), assessed by dual $\mathrm{x}$-ray absorptiometry, were both higher in 20-week-old RXR-KO male mice than in their WT littermates (Figure 1, A and B). $\mu \mathrm{CT}$ and bone histomorphometry showed increased cortical and trabecular bone volume in RXR-KO femurs from 20-week-old mice (Figure 1, C and D). However, no significant differences were detected in any of these parameters between WT and RXR-KO mice at 8 weeks (Supplemental Figure 2). We also found that 20-week-old female RXR-KO mice displayed no bone abnormalities (Supplemental Figure 3). These findings show that bone mass is specifically increased in RXR-KO male mice and that this phenotype becomes pronounced as the mice age. We next assessed osteoblast and osteoclast function. Osteoblasts have been shown to express Cre recombinase in Mx1-Cre transgenic mice (13); however, neither osteoblast number (Supplemental Figure 4, A and B) nor activity (Supplemental Figure 4, C-F) was affected in RXR-KO male mice. On the other hand, osteoclasts in RXR-KO male mice were enlarged (Figure 1, E-G). Transmission electron microscopy (TEM) revealed that WT osteoclasts displayed cytoplasmic vacuolization and a ruffled border and were attached to the bone matrix (Figure $1 \mathrm{H}$ ). These are features of normally activated, mineral-resorbing osteoclasts (14). In contrast, osteoclasts in RXR-KO mice had partial or disorganized ruffled borders and were in limited contact with the bone matrix (Figure $1 \mathrm{H}$ ), suggestive of defective lytic activity (15). Consistently, RXR-KO male mice presented low levels of the osteoclast activity markers tartrate-resistant acid phosphatase (TRAP) and C-terminal telopeptide (CTX) in serum and deoxypyridinoline (DPD) in urine (Figure 1I). In addition, the total bone mRNA levels of osteoclastactivity-related genes (such as nuclear factor of activated T cells, cytoplasmic, calcineurin dependent 1 [Nfatc1], TRAP 5 [Acp5], and carbonic anhydrase 2 [Car2]) were lower in RXR-KO mice than in WT (Supplemental Figure 4G). These findings indicate that the lack of RXRs in hematopoietic cells increases bone mass in male mice by impairing osteoclast activity.

$R X R-K O$ mice are protected from ovariectomy-induced bone loss. Postmenopausal osteoporosis is a prevalent bone pathology that develops as a result of increased osteoclast differentiation and activity (7). To model the potential role of RXRs in bone homeostasis under this pathological condition, we induced estrogen deficiency by bilateral ovariectomy (OvX) in 20-week-old WT and RXR-KO mice and assessed bone loss over a further 8 weeks. Consistent with the lack of a skeletal phenotype in RXR-KO females (Supplemental Figure 3), age- and sex-matched sham-operated WT and RXR-KO mice showed no differences in bone architecture or osteoclast activity (Figure 2). However, after OvX, loss of trabecular bone mass was significantly lower in RXR-KO mice (Figure 2, A-D). In WT mice, OvX led to a marked increase in osteoclast activity, shown by a rise in urine DPD and plasma CTX concentrations (Figure 2, E and F). In contrast, the increase in DPD and CTX concentrations was significantly lower in ovariectomized RXR-KO mice (Figure 2, E and F). These findings indicate that the diminished osteoclast activity in RXR-KO female mice makes them less prone to bone loss upon OvX.

Lack of RXRs leads to formation of giant and nonresorbing osteoclasts in vitro. To determine the mechanism underlying the abnormal osteoclast activation in RXR-KO mice, we cultured bone marrow cells in the presence of the cytokines macrophage colony-stimulating factor (M-CSF) and RANKL (16). Osteoclasts differentiated from RXR-KO bone marrow were significantly larger than WT-derived osteoclasts and contained more nuclei (Figure 3, A-C). In contrast, osteoclast differentiation from bone marrow cells lacking $\mathrm{RXR} \alpha$ but not RXR $\beta$ was normal, supporting the functional redundancy of $\operatorname{RXR} \alpha$ and $R X R \beta$ in hematopoietic cells (Supplemental Figure 5). Enlargement of osteoclasts can result in increased bone resorption (17); however, the abnormally large RXR-KO osteoclasts displayed low lytic activity when cultured in calcium-phosphate-coated plates (Figure 3D) or on bovine cortical bone slices (Figure 3E). RXR-KO osteoclasts also showed low expression of Acp5, matrix metallopeptidase 9 (Mmp9), cathepsin-K (CtsK), and Car2, which encode molecules crucial for extracellular matrix degradation and bone resorption (Figure $3 \mathrm{~F}$ ). In addition, real-time imaging showed that RXR-KO osteoclasts had an increased spreading over the substrate compared with WT osteoclasts (Figure 3G and Supplemental Video 1). These in vitro results are consistent with our in vivo observations, indicating that the lack of RXRs in osteoclast progenitors leads to a cell-autonomous effect on osteoclast differentiation and activation.

The M-CSF response is altered in RXR-KO osteoclast progenitors. Osteoclast size is determined by the response of osteoclast progenitors to $\mathrm{M}-\mathrm{CSF}$, which controls their proliferation and survival $(18,19)$. This cytokine also stimulates activities of the mature resorptive osteoclast, such as spreading, motility, and cytoskeletal organization (20). We thus hypothesized that the RXR-KO osteoclast phenotype could be due to an altered M-CSF response. To test this, we first characterized the capacity of osteoclast progenitors to form myeloid colony-forming units (CFUs) and to divide in response to M-CSF. After 12 days in the presence of M-CSF, the same numbers of CFUs were seen in WT and RXRKO bone marrow cultures (Figure 4A); however, the number of cells per colony was significantly higher in RXR-KO CFUs (Figure $4 \mathrm{~B})$. In addition, a higher percentage of osteoclast progenitors from RXR-KO mice proliferated in response to M-CSF (Figure $4 \mathrm{C}$ ). RXR deletion had no effect on myeloid progenitor or monocyte numbers, which indicates that the differences between the WT and the RXR-KO responses to M-CSF were due to a higher 

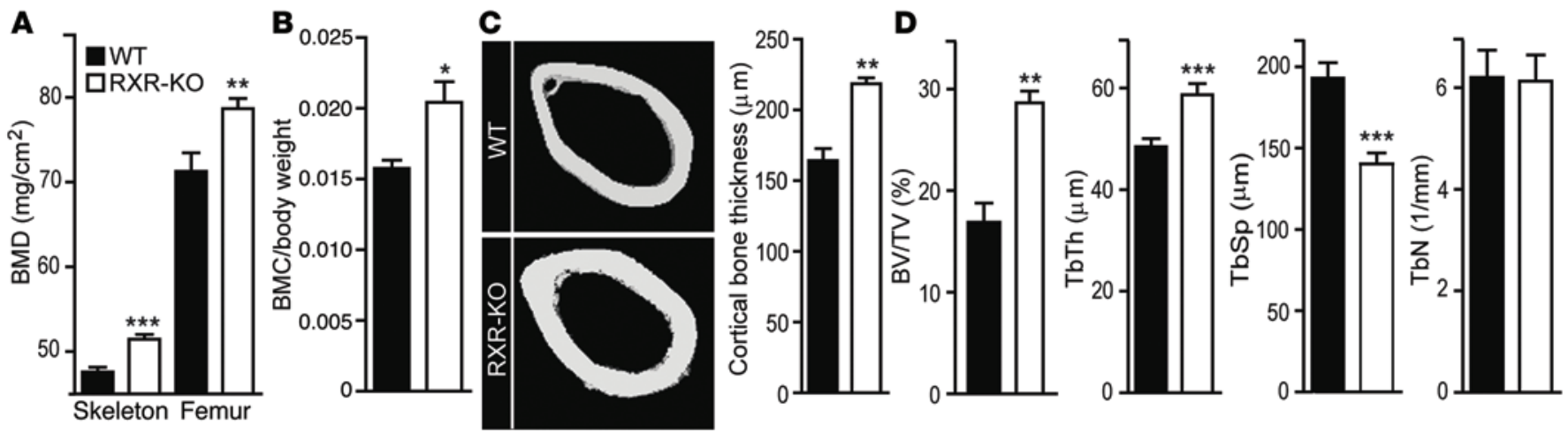

$\mathbf{E}$

WT

RXR-KO
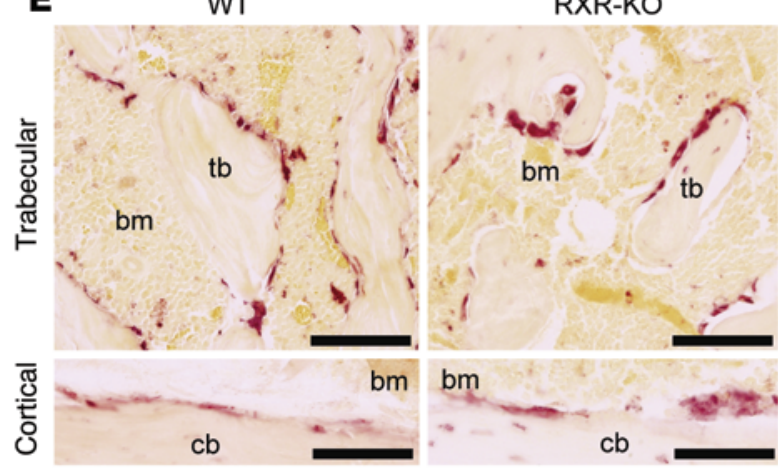

H

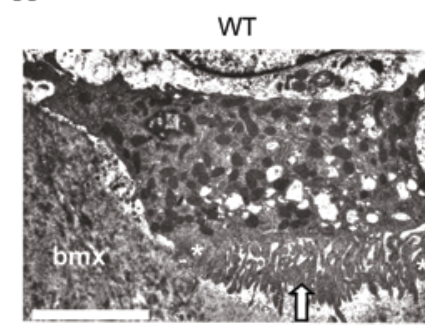

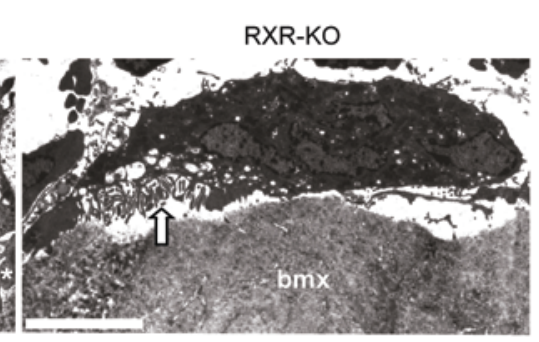

$\mathbf{F}$

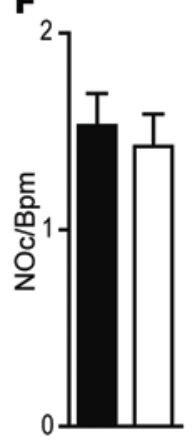

G
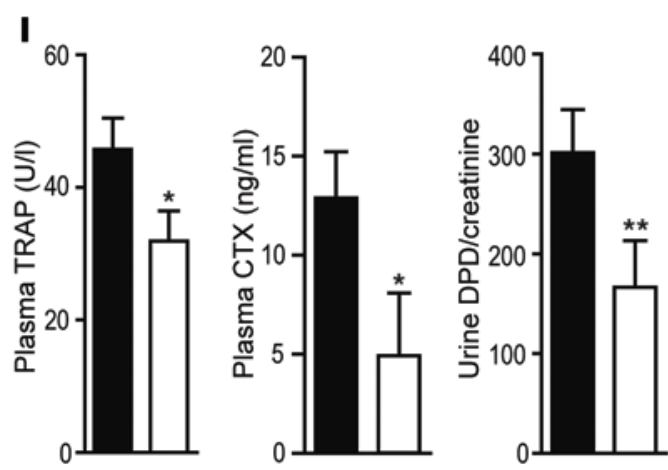

Figure 1. Increased bone mass and reduced osteoclast activity in 20-week-old RXR-KO male mice. (A and B) BMD and BMC measured by DEXA; BMC values are normalized to body weight $(\mathrm{g} / \mathrm{g}) . n=8$ mice per genotype. (C) Representative $\mu C T$ images showing cortical bone of the femoral shaft and cortical bone thickness measured on $\mu$ CT scans. $n=5$ mice per genotype. (D) Histomorphometric analysis of the tibial end of the femur. BV/TV (\%), relative trabecular bone volume; TbTh, trabecule thickness; TbSp, trabecule separation; TbN, trabecule number. $n=8$ per genotype. (E) TRAP staining of the tibial end of the femur. bm, bone marrow; tb, trabecule; cb, cortical bone. Scale bars: $85 \mu \mathrm{m}$. (F and $\mathbf{G})$ Osteoclast number $(\mathrm{NOc} / \mathrm{Bpm})\left(\mathrm{mm}^{-1}\right)$ and surface $(\mathrm{OcS} /$ $\mathrm{Bs})(\%)$ in femur sections, normalized to bone perimeter (Bpm) and bone surface (BS). $n=6$ per genotype. (H) Representative TEM images of osteoclasts in the femur from 2 independent studies using 3 mice per genotype. bmx, bone matrix. Arrows show ruffled border; asterisks indicate the attachment zone. Scale bars: $5 \mu \mathrm{m}$. (I) Clinical chemistry of osteoclast activity. $n=9$ (TRAP, DPD) and 6 (CTX) per genotype. Data are presented as mean \pm SEM. ${ }^{*} P<0.05$; ${ }^{* *} P<0.01 ;{ }^{* *} P<0.001$, compared with WT (unpaired 2-tailed Student's $t$ test).

proliferation rate of the RXR-KO progenitors rather than to a difference in the number of osteoclast progenitors (Supplemental Figure 6). Next, to determine whether the RXR-KO osteoclasts had altered cytoskeletal organization, we cultured osteoclasts on glass coverslips or bovine cortical bone slices and examined the organization of F-actin and the distribution of Wiskott-Aldrich syndrome protein (WASP) by confocal immunofluorescence. In WT osteoclasts plated on glass coverslips or bone slices, F-actin and WASP were seen in podosomes, arranged in dense podosome belts or actin rings (the sealing zone), respectively (Figure 4, D and E). This podosome arrangement is a hallmark of mature, resorbing osteoclasts (21-23). In contrast, most RXR-KO osteo- clasts presented scattered podosomes, failing to form a morphologically normal podosome belt on the glass surface or a sealing zone on bone (Figure 4, D and E).

All these findings suggest that the giant, resorption-deficient phenotype of RXR-KO osteoclasts is due to a defective response to $\mathrm{M}-\mathrm{CSF}$, affecting osteoclast progenitor proliferation as well as the organization and maturation of cytoskeletal structures required for bone resorption.

Mafb expression is diminished in RXR-KO osteoclast progenitors. To determine the molecular mechanism underlying the abnormal RXR-KO osteoclast phenotype, we measured the transcript levels of RANKL- and M-CSF-responsive genes in the course of 
A
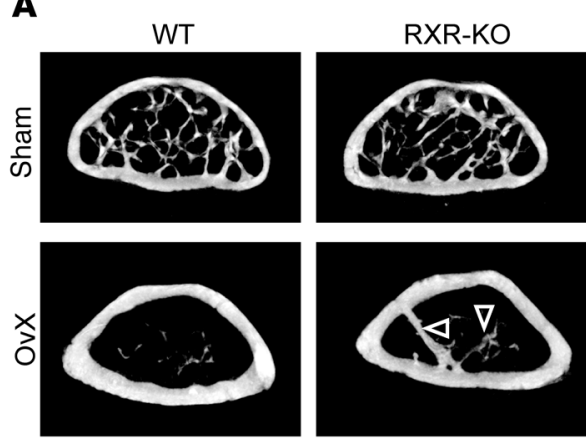

B
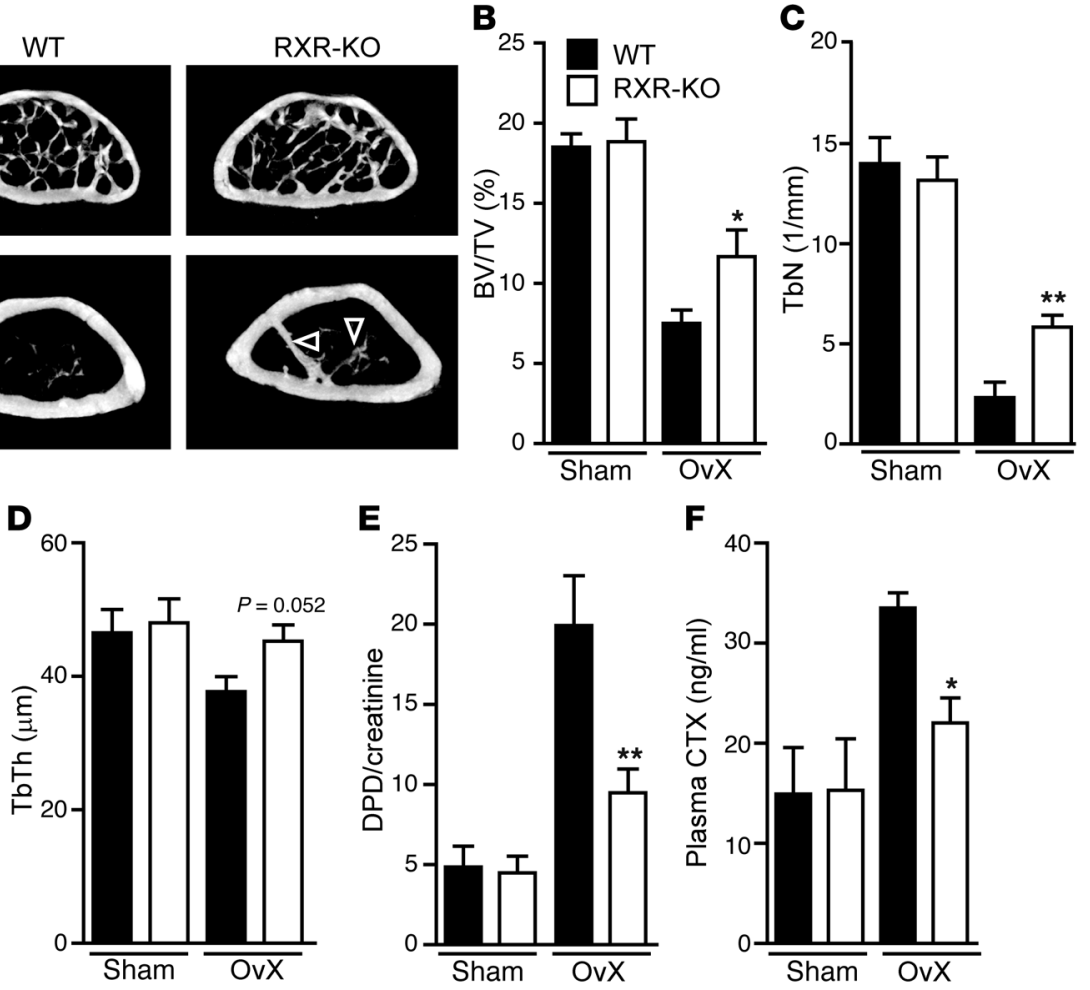

F

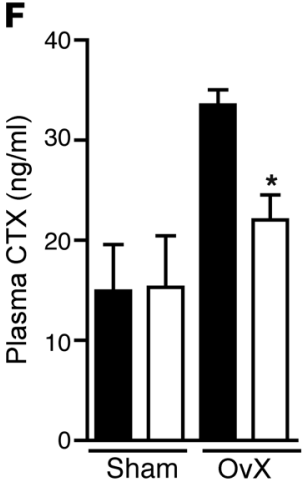

Figure 2. Therapeutic potential of RXR deletion in a model of postmenopausal osteoporosis. (A) Representative $\mu \mathrm{CT}$ images of 3 mice per genotype, showing the femur $1 \mathrm{~mm}$ below the distal epiphysis; arrowheads point to bone trabecules. (B-D) Bone histomorphometry of the femur. $n=6$ per genotype. (E and $\mathbf{F}$ ) Levels of plasma and urine osteoclast activity markers. $n=6$ per genotype. 20-week-old female mice were used in the experiments; data are presented as mean \pm SEM. ${ }^{*} P<0.05 ;{ }^{* *} P<0.01$, compared with ovariectomized WT mice (unpaired 2-tailed Student's $t$ test).

(Figure 5G). Next, to assess whether low MAFB expression in osteoclast progenitors recapitulates the osteoclast phenotype of RXR-deficient progenitors, we silenced Mafb in bone marrow cells by RNA interference. Mafb downregulation in osteoclast progenitors (Supplemental Figure 8D) resulted in larger osteoclasts (Figure 5H and Supplemental Figure 8E) with increased nuclear number (Figure 5I), decreased transcription of Ctsk, a gene encoding an

osteoclast differentiation in vitro. Upon treatment with M-CSF and RANKL, WT and RXR-KO cells showed indistinguishable transcriptional responses for the transcription factor Spi1 (PU.1, involved in the formation and survival of osteoclast precursors), cytokine receptors (CsfIr and Tnfrsf11a), fusion proteins (Dcstamp and Atp6vOd2), and adhesion molecules (Src and Itga3) (Supplemental Figure 7). These results excluded changes in the expression of cell fusion and adhesion molecules as the cause of the giant and resorption-deficient RXR-KO osteoclast phenotype. The transcription factors Mafb, Fos, Nfatclc, and Mitf and the receptors Oscar and Calcr, all regulated by RANKL signaling in WT cells (24), were not modulated throughout the course of osteoclast differentiation in RXR-KO cells (Figure 5A and Supplemental Figure 7). However, of these transcription factors, only MAFB showed significantly lower expression (mRNA and protein) in RXR-KO osteoclast progenitors than in WT (Figure 5, A-C). Low Mafb expression was also found in RXR-KO bone marrow early blasts, myeloid blasts, and monocytes, all considered osteoclast progenitors (25) (Figure 5D and Supplemental Figure 1D). These results identify MAFB as the main molecule differentially expressed between WT and RXR-KO osteoclast progenitors.

Interestingly, MAFB deficiency has been previously shown to enhance sensitivity of hematopoietic progenitors and myeloid cells to M-CSF, influencing their proliferation and survival $(26,27)$. To clarify whether an RXR/MAFB axis is established in osteoclast progenitors with a role in proliferation, we next performed MAFB gain-of-function experiments in RXR-KO osteoclast progenitors. Lentivirus-mediated overexpression of MAFB in RXR-KO cells (Supplemental Figure 8, A and B) restored the WT response to M-CSF, both in the number of cells per CFU (Figure 5, E and F, and Supplemental Figure $8 \mathrm{C}$ ) and the percentage of proliferation important protease that degrades collagen and other matrix proteins during bone resorption (Figure $5 \mathrm{~J}$ and ref. 13), and decreased lytic activity (Figure 5K). Together, these results demonstrate that the abnormal osteoclast proliferation, differentiation, and activity observed in RXR-KO cells is likely to be dependent on MAFB expression in osteoclast progenitors.

RXR homodimers regulate Mafb transcription in basal physiological conditions. We next determined whether Mafb is a bona fide RXR target gene. Treatment with RXR pan agonists (LG100268 [LG268] and 9-cis-retinoic acid [9cRA]) induced Mafb gene expression in WT osteoclast progenitors (Figure 6A). Interestingly, both basal and RXR agonist-induced Mafb expression were downregulated by LG100754 (LG754), an agonist of RXR/peroxisome proliferator-activated receptor (PPAR) and RXR/retinoic acid receptor (RAR), but an antagonist of RXR homodimers (ref. 28 and Figure 6A). The MAFB induction (mRNA and protein) by RXR agonists in WT osteoclast progenitors was not observed in RXR-KO cells, indicating that the effect is RXR dependent (Figure 6, B and C). In a transfection assay in the LXR-, PPAR-, and RXRdeficient cell line RAW264.7 (ref. 29 and Supplemental Figure 9, A and B), the activity of a 1.5-kilobase Mafb promoter was upregulated by LG268 and 9cRA and downregulated by LG754 only in the presence of overexpressed RXR $\alpha$ (Figure 6D). Moreover, LG754 inhibited 9cRA-induced Mafb promoter activation (Supplemental Figure 9C). These data suggest that Mafb is a target gene of RXR homodimers. Using luciferase reporter assays of truncated Mafb promoters, ChIP followed by high-throughput sequencing (ChIPseq), and motif discovery analysis, we identified 2 RXR-binding motifs (DR-1) in the Mafb promoter (Figure 6E and Supplemental Figure 9, D and E). In vivo recruitment of RXR to the Mafb promoter was further investigated by ChIP in osteoclast progenitors. 
A

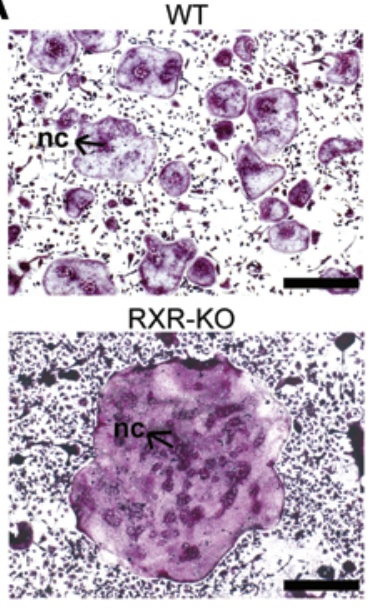

B

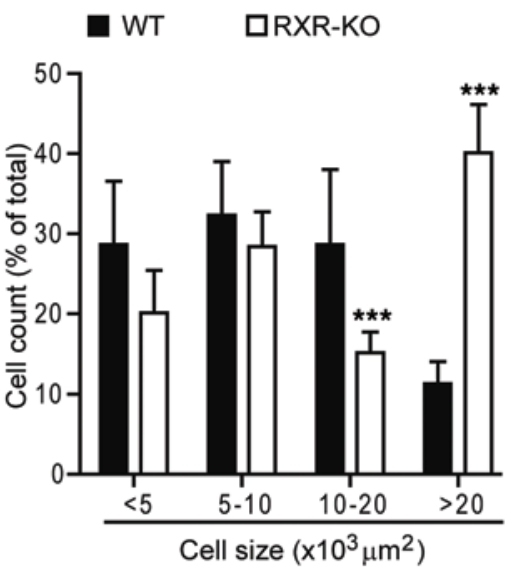

C

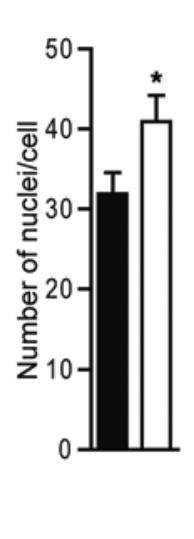

D

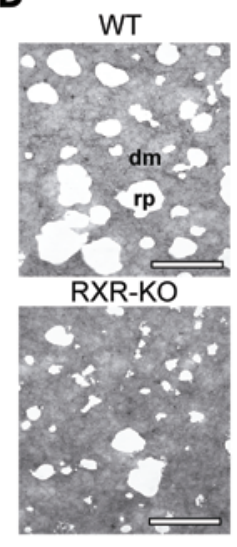

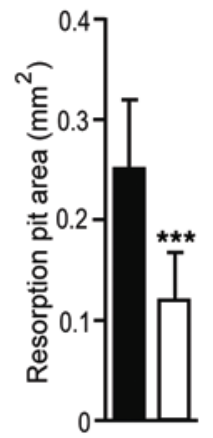

E

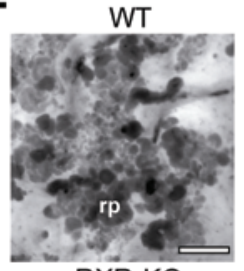

RXR-KO

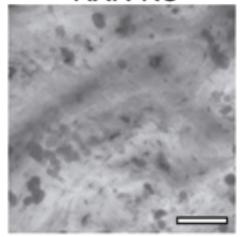

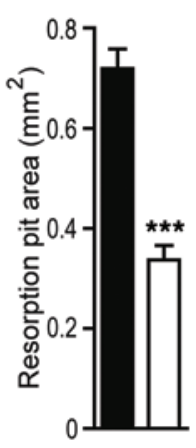

$\mathbf{F}$

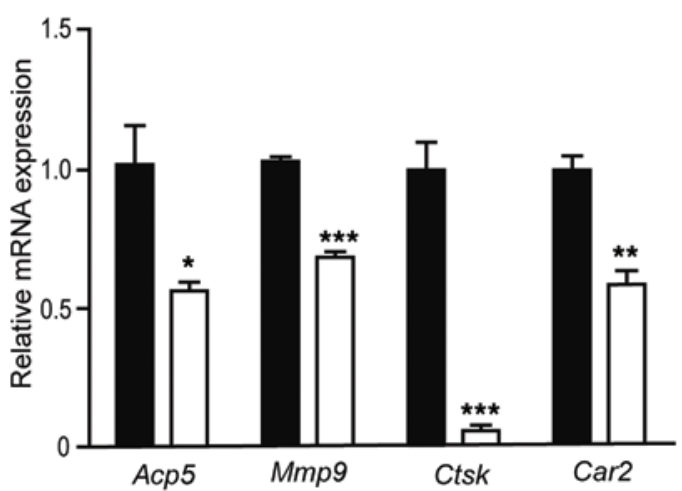

G

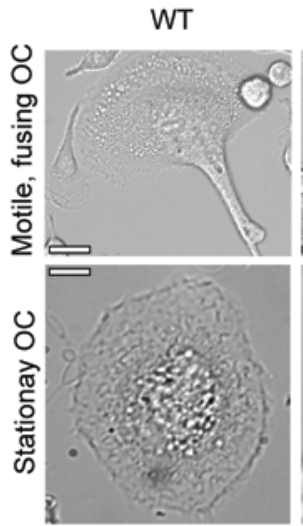

RXR-KO

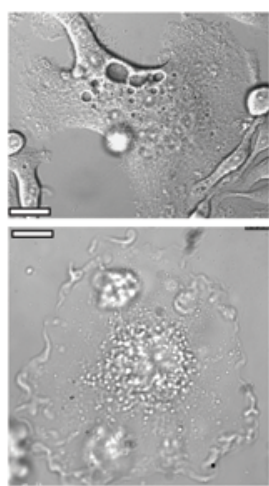

Figure 3. Giant and nonresorbing osteoclasts develop in the absence of RXRs. (A) TRAP-positive osteoclasts differentiated from bone marrow of WT and RXR-KO mice. nc, nuclei. Scale bars: $100 \mu \mathrm{M}$. (B and C) Cell size and number of nuclei in the cultured TRAP-positive osteoclasts. (D) Resorption activity of osteoclasts was measured by plating them on calcium-phosphate-coated plates; the remaining matrix (dm) is stained with Toluidine Blue; area of resorption pits (rp) was quantified. Scale bars: $100 \mu \mathrm{m} . n=6$ per genotype. (E) Resorption activity of osteoclasts on bovine cortical bone slices; the resorption pits were stained with Toluidine Blue, and the resorption pit area was quantified. Scale bars: $100 \mu \mathrm{m} . n=6$ per genotype. (F) Relative mRNA expression of osteoclast activity genes in in vitro-differentiated osteoclasts. $n=3$ per genotype. (G) DIC images showing motile and stationary osteoclasts (OC) in vitro (see Supplemental Video 1). Data are presented as mean \pm SEM. ${ }^{*} P<0.05 ;{ }^{* *} P<0.01$; ${ }^{* *} P<0.001$, compared with WT (unpaired 2-tailed Student's $t$ test). $\mathbf{A}-\mathbf{C}$ and $\mathbf{F}$ show representative experiments of 3 done in triplicate.

RXR bound to the proximal promoter of Mafb in basal conditions in WT but not in RXR-KO cells, and its binding was further enhanced by RXR ligand activation (Figure 6F). Since DR-1 motifs are also binding sites for PPAR $\gamma(30)$, we next analyzed whether PPAR $\gamma$ regulates Mafb in basal conditions. No binding of PPAR $\gamma$ was detected in the ChIP-seq assays (Supplemental Figure 9D), and we observed no differences in Mafb mRNA levels in PPAR $\gamma$ deficient osteoclast progenitors (Pparg-KO) compared with WT cells (Supplemental Figure 9F). Together, these results show that RXR binds to DR-1 sequences of the Mafb promoter in osteoclast progenitors. This, along with the low MAFB expression found in osteoclast progenitors of RXR-KO mice (Figure 5, A-D), demonstrates the existence of an RXR homodimer/MAFB regulatory axis under basal physiological conditions.

Pharmacological activation of RXRs blocks osteoclast differentiation through RXR/LXR-mediated upregulation of Mafb. We next assessed the effect of pharmacological RXR activation on $\mathrm{M}-\mathrm{CSF}-$ and RANKL-induced osteoclast differentiation in vitro.
Unexpectedly, when cells were treated with LG268, osteoclast differentiation from WT but not from RXR-KO bone marrow cells was abolished (Figure 7A). Measurement of Mafb transcript levels in the course of osteoclast differentiation showed that WT but not RXR-KO cells upregulated Mafb expression at day 5 of in vitro differentiation (Figure 7B). This Mafb upregulation can account for the blockade of osteoclast differentiation in WT cells, since MAFB downregulation in differentiating osteoclasts is required for functional osteoclast maturation (31).

To assess whether induction of Mafb by pharmacological activation of RXR is mediated by its heterodimerization with another nuclear receptor, we treated osteoclast progenitors with agonists for all the RXR heterodimeric partners expressed by myeloid cells (8). We observed that only LXR-specific agonists (GW3965 and T0901317 [T1317]) induced Mafb expression (Supplemental Figure 10, A and B). Using RXR-KO and Lxr-KO osteoclast progenitors, we found that LXR agonist-mediated induction of MAFB mRNA and protein expression was dependent on RXR and LXR expression 

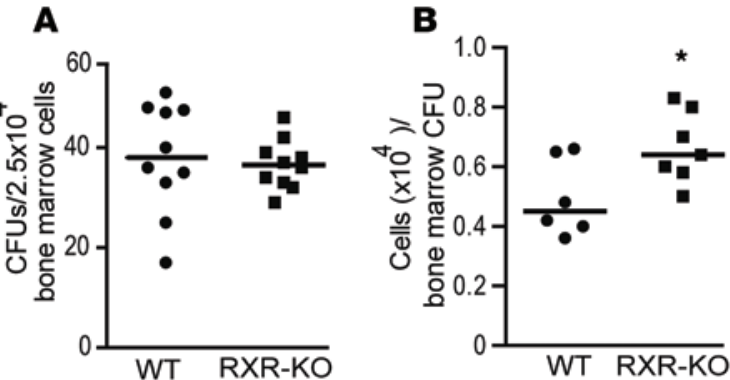

D

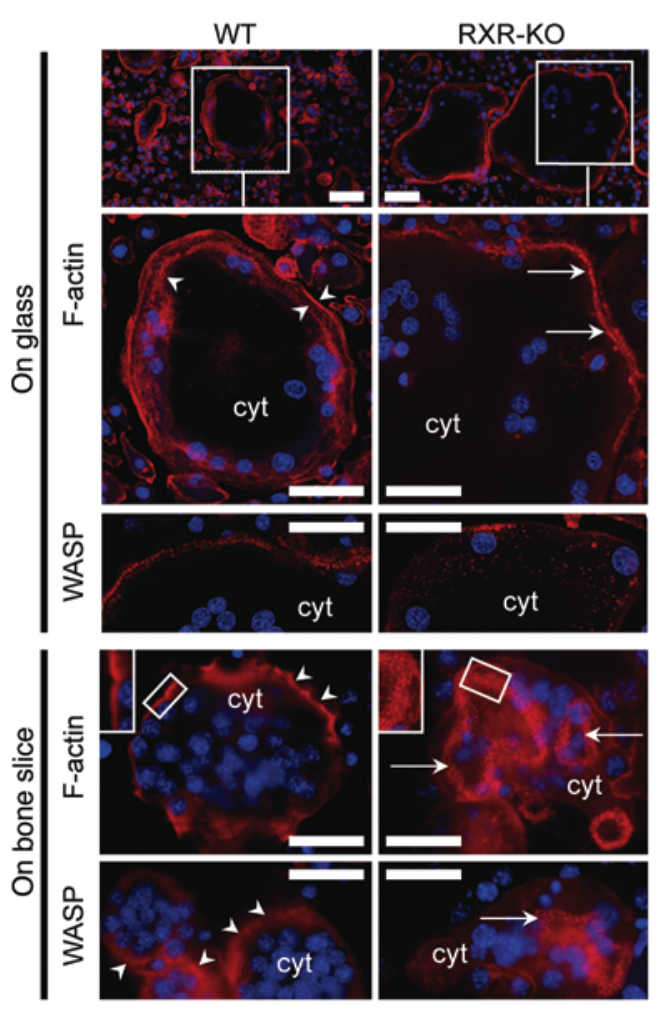

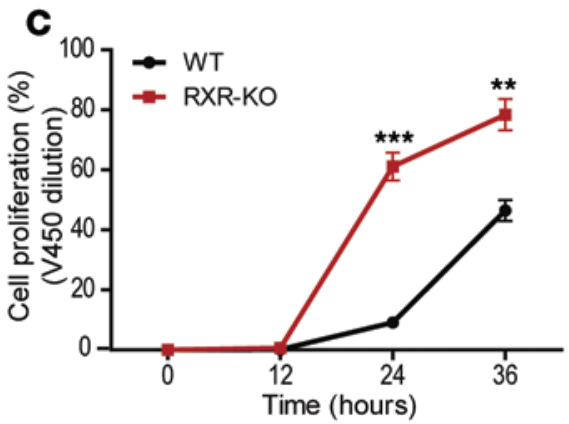

E
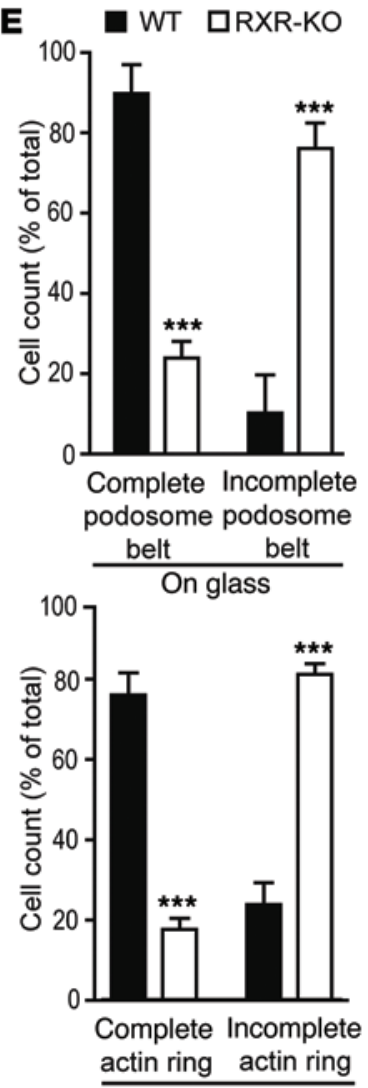

On bone slice
Figure 4. Altered RXR-KO osteoclast progenitor proliferation and cytoskeletal organization in response to M-CSF. (A and B) CFU assay in bone marrow: CFU number (A) and number of cells per CFU (B) in WT and RXR-KO osteoclast progenitor cultures. $n=6-10$ per genotype. (c) Flow cytometry analysis of the proliferative responses of WT and RXRKO bone marrow osteoclast progenitors (representative of 3 independent experiments done in triplicate). (D) Phalloidin labeling of F-actin and WASP staining of osteoclasts cultured on glass coverslips or bovine cortical bone slices. cyt, cytoplasm. Arrows show podosomes; arrowheads indicate podosome belts (on glass) and actin rings (on bone). Scale bars: $25 \mu \mathrm{m}$. (E) Percentage of cultured osteoclasts with a complete podosome belt or actin ring or with incomplete actin ring and scattered podosomes (representative experiment $[n=3-7$ replicates] of 2-3 performed). $\mathbf{C}$ and $\mathbf{E}$ are presented as mean \pm SEM. ${ }^{*} P<0.05 ;{ }^{* *} P<0.01$; ${ }^{* * *} P<0.001$, compared with WT, using unpaired 2-tailed Student's $t$ test (C) or 2-tailed Mann-Whitney $U$ test $(\mathbf{E})$.
(Figure 7, C-E). Supporting this, Mafb reporter activity was induced by LG268 and T1317 only in the presence of overexpressed RXR and LXR (Figure 7F). Positive regulation of target genes by RXR/ LXR often involves the binding of the heterodimer to a liver $\mathrm{X}$ response element (LXRE). However, no LXR binding to the Mafb promoter was detected in the ChIP-seq analysis (Supplemental Figure 9D). These data suggested that RXR/LXR may not regulate Mafb expression by a classic LXRE-binding mechanism and that, instead, additional LXR-dependent pathways may be involved. In accordance with this, we found that the mRNA and protein expression of the transcription factor SREBP-1c were induced by RXR/ LXR activation in WT osteoclast progenitors, but not in RXR-KO (Figure 8, A and B) or Lxr-KO cells (Supplemental Figure 10C). Accordingly, we found that $M a f b$ reporter activity was enhanced in the presence of transiently overexpressed SREBP-1c (Figure 8C). In addition, using luciferase assays, ChIP-seq assays, and motif analysis, we determined the existence of 3 SREBP-1c-binding sites along the Mafb proximal promoter (Figure 8C, Supplemental Fig- ure 9D, and Supplemental Figure 10D). In vivo binding of SREBP1c to the Mafb promoter was further confirmed by ChIP analysis in osteoclast progenitors (Figure 8D). Finally, Srebp1c silencing blocked Mafb induction by T1317, confirming that the RXR/LXRdependent increase in $M a f b$ expression was mediated by SREBP-1c (Figure 8, E and F).

We next assessed the effect of LXR pharmacological activation on osteoclast differentiation. Treatment of in vitro differentiating osteoclasts with T1317 induced Mafb expression at day 5 of differentiation and accordingly blocked osteoclast differentiation in WT but not in RXR- or LXR-deficient cells (Supplemental Figure 11). It was notable that, in contrast to RXR-KO cells, basal mRNA and protein MAFB expression levels in osteoclast progenitors from WT and $L x r-\mathrm{KO}$ mice were similar (Figure 7, $\mathrm{D}$ and E). Accordingly, there was no size difference between osteoclasts differentiated from WT or Lxr-KO osteoclast progenitors in the absence of any ligand treatment (Supplemental Figure 11B). These results support the idea that MAFB expres- 
A

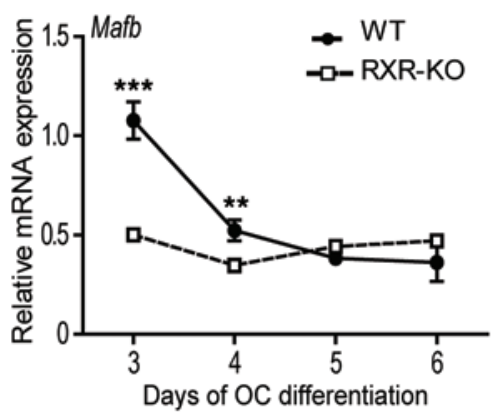

E

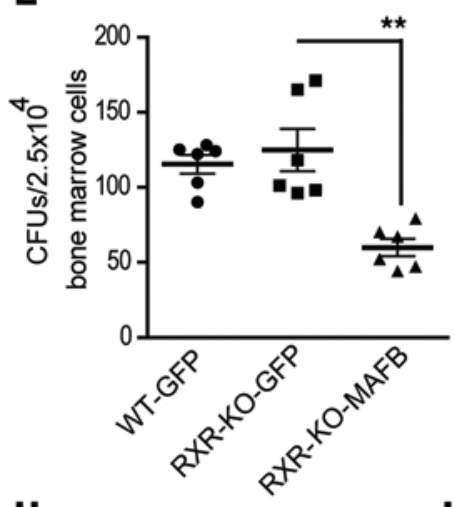

H

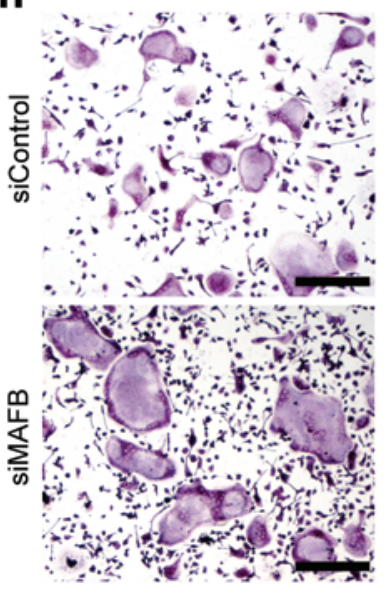

B

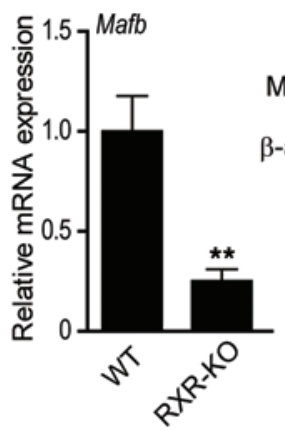

$\mathbf{F}$

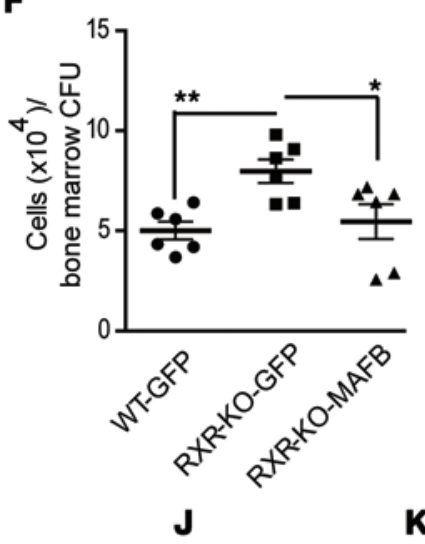

D

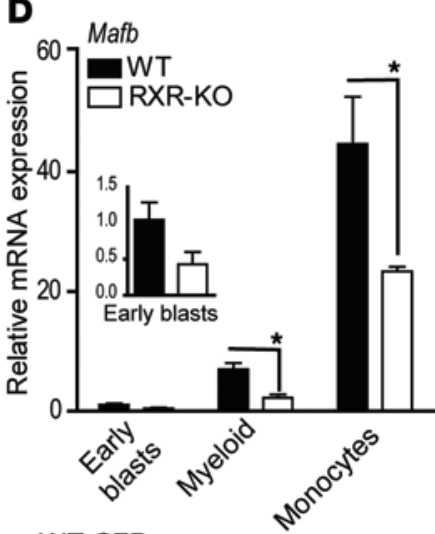

G

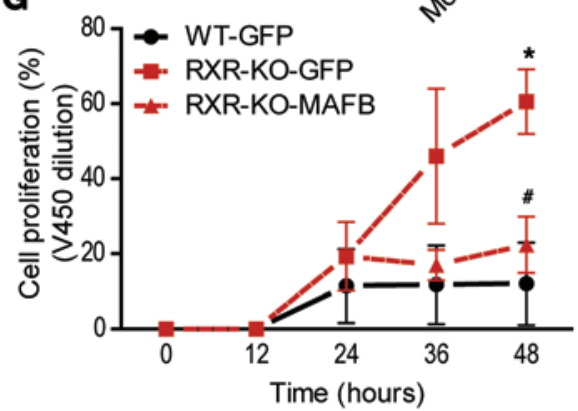

I

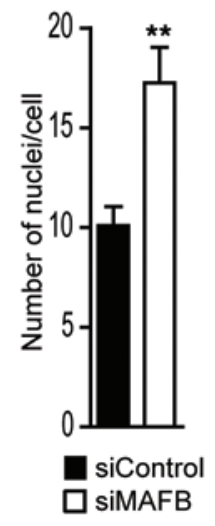

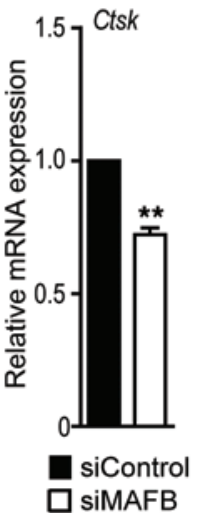

$\mathbf{K}$

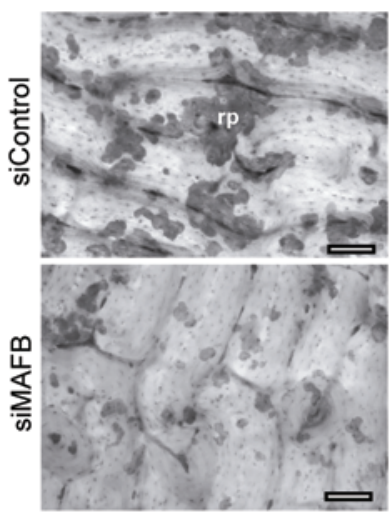

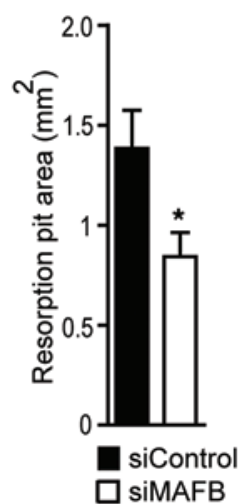

Figure 5. Low MAFB expression in osteoclast progenitors underlies the abnormal RXR-KO osteoclast phenotype. (A and B) Relative mRNA expression of Mafb in the course of osteoclast differentiation in vitro (A) and in osteoclast progenitors (B). ${ }^{* *} P<0.01$; ${ }^{* *} P<0.001$, compared with RXR-KO cells on the same day of differentiation. (C) MAFB protein expression in osteoclast progenitors (representative of 3 mice per genotype). (D) Mafb mRNA expression in bone marrow myeloid populations; cells were isolated as shown in Supplemental Figure 1D. (E-C) Proliferation assays in lentiviral-mediated MAFB-overexpressing RXR-KO osteoclast progenitors: number of CFUs (E) and number of cells per CFU (F) in bone marrow cell cultures infected with control lentivirus (WT-GFP and RXR-KO-GFP) and with MAFB lentivirus (RXR-KO-MAFB). $n=6$ per group. (C) Flow cytometry analysis of the proliferative responses of osteoclast progenitors infected with control lentivirus (WT-GFP and RXR-KO-GFP) and with MAFB lentivirus (RXR-KO-MAFB). $n=3$ per group. ${ }^{*} P<0.05$, compared with WT-GFP; ${ }^{P} P<0.01$, compared with RXR-KO-GFP. (H-K) siRNA assay: representative TRAP-positive cells (H), number of nuclei (I), Ctsk expression (J), and resorption pit area (K) in osteoclast cultures after 5 days of differentiation from bone marrow cells transfected with control or Mafb siRNAs. ${ }^{*} P<0.05$; ${ }^{* *} P<0.01$, compared with siControl. Resorption activity was measured after culturing day-5 osteoclasts on bone bovine cortical bone slices for 2 additional days; the experiment shown is representative of 3 independent experiments done in triplicate. Scale bars: $100 \mu \mathrm{m}$. Data are presented as mean \pm SEM. Statistical comparisons were made by unpaired 2-tailed Student's $t$ test.

sion under basal physiological conditions is regulated by RXR homodimers, whereas, during osteoclastogenesis, pharmacological activation of RXR, through RXR heterodimerization with LXR and induction of SREBP-1c, induces MAFB expression and blocks osteoclast differentiation.
Protective effect of pharmacological RXR activation on bone loss. To investigate the effect of pharmacological RXR activation on osteoclasts and bone resorption in vivo, we treated 8-week-old C57BL/6 male mice with the RXR ligand bexarotene for 6 weeks. Bexarotene did not affect bone parameters (Supplemental Figure 

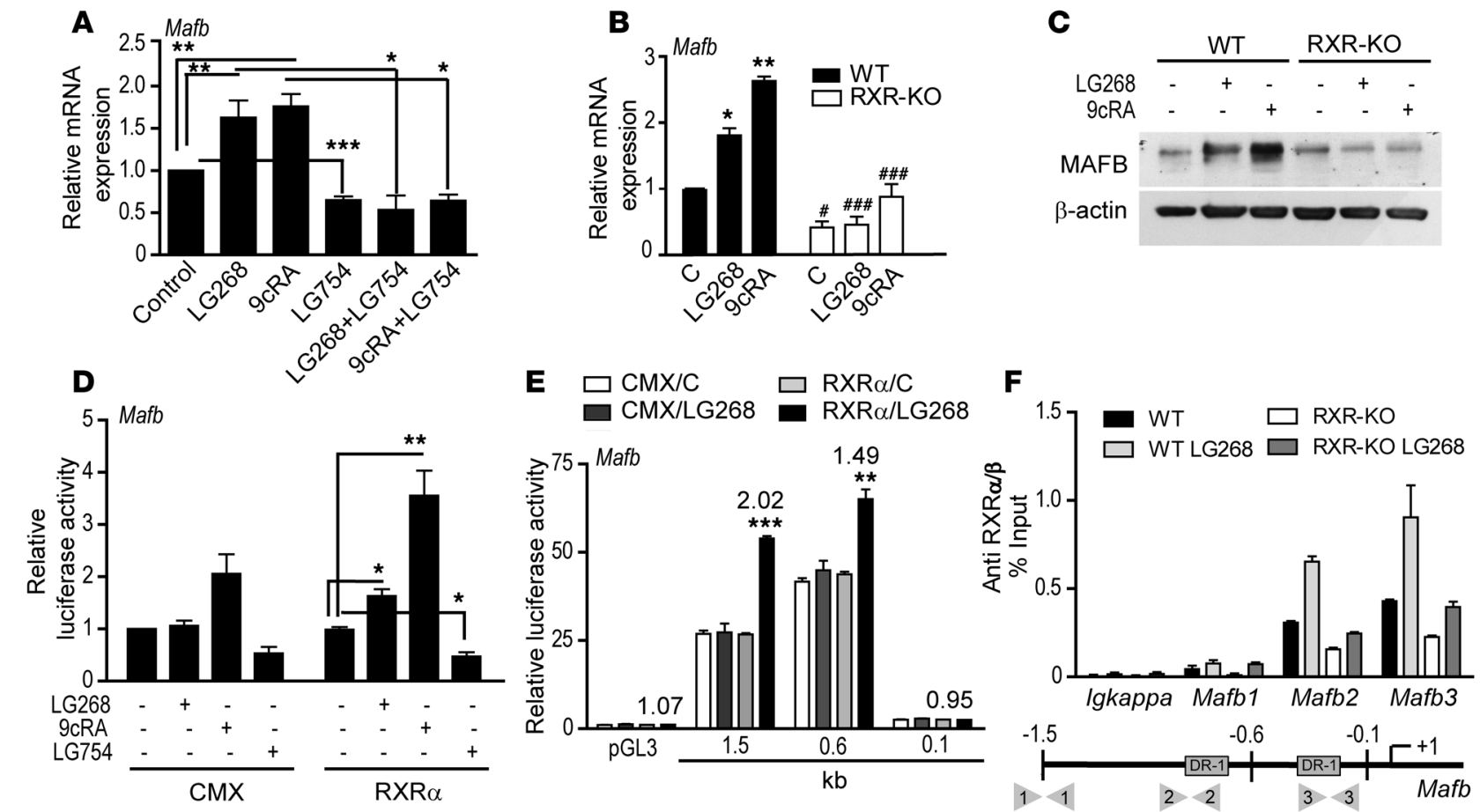

Figure 6. RXR homodimers regulate Mafb expression in osteoclast progenitors. (A) Mafb mRNA expression in WT osteoclast progenitors treated with the RXR agonists LC268 and 9CRA and with the antagonist of RXR homodimers LG754. Mafb mRNA (B) and protein (C) expression in WT and RXR-KO osteoclast progenitors treated with LG268 or 9cRA. ${ }^{*} P<0.05$; ${ }^{* *} P<0.01$, compared with WT vehicle-treated cells (C) (paired 2-tailed Student's $t$ test). ${ }^{\#} P<0.05 ;{ }^{\#} P<0.01$, versus the equivalent treatment in WT cells (unpaired 2-tailed Student's $t$ test). (D and E) Luciferase reporter assays in RAW264.7 cells transfected with (D) RXR $\alpha$ or CMX empty vector together with a reporter vector containing $1.5 \mathrm{~kb}$ of the Mafb promoter or (E) the indicated Mafb promoter and pGL3 control reporters. Data are relative values compared with the vehicle-treated Mafb reporter (D) or the vehicle-treated pGL3 reporter (E) in the absence of RXR $\alpha$; in $\mathbf{E}$, the indicated fold inductions represent $(\mathrm{RXR} \alpha / \mathrm{LG} 268) /(\mathrm{RXR} \alpha / \mathrm{C}) .{ }^{*} P<0.01$; ${ }^{* * *} P<0.001$, compared with vehicletreated reporter in the presence of RXR $\alpha$. (F) ChIP analysis of RXR $\alpha / \beta$ binding to $-1.5 \mathrm{~kb}$ (Mafb1), $-1.0 \mathrm{~kb}$ (Mafb2), and $-0.4 \mathrm{~kb}$ (Mafb3) regions of the Mafb promoter or to a negative control (Igkappa) in osteoclast progenitors. Lower panel, localization of RXR-binding sites in the Mafb promoter. Arrows indicate the position of primers used for qPCR. Experiment shown is representative of 3 done in triplicate. Data are presented as mean \pm SEM ( $n=3$ per group). ${ }^{*} P<0.05 ;{ }^{* *} P<0.01$; ${ }^{* * *} P<0.001$, by paired 2 -tailed Student's $t$ test $(\mathbf{A}, \mathbf{D}$, and $\mathbf{E}$ ). $\mathbf{C}$ is representative of 3 independent experiments.

12, A and B) and had no significant effect on osteoclast (Supplemental Figure 12C) or osteoblast (Supplemental Figure 12D) activity. These findings show that bexarotene does not affect osteoclastmediated bone resorption during steady-state bone turnover.

Next, to evaluate the therapeutic potential of pharmacological activation of RXRs in the treatment of pathologic bone loss, we performed OvX in 20-week-old female mice and treated them with bexarotene. After 8 weeks of bexarotene treatment, sham-operated mice showed no differences in bone parameters and osteoclast activity compared with vehicle-treated controls (Figure 9). In contrast, in ovariectomized mice, bexarotene treatment significantly increased the bone volume and the trabecule number, as shown by $\mu \mathrm{CT}$ and bone histomorphometric analysis (Figure 9, A-C). This increase in bone mass correlated with an inhibition of osteoclast activity, determined by the lower urine DPD/creatinine ratio (Figure 9D), and a decrease in mean levels of serum CTX (Figure 9E) in bexarotenetreated ovariectomized mice. These findings show that pharmacological RXR activation has a protective effect against bone loss after OvX by diminishing osteoclast activation and bone resorption.

\section{Discussion}

Classic antiresorptive therapies act by reducing osteoclast viability (7). As a consequence, paracrine signaling from osteoclasts to osteoblasts is disrupted, which can result in suppression of osteoblast-driven bone formation (7, 32). New therapies are therefore focused on compounds that inhibit osteoclast activity rather than their viability, so that physiological communication between osteoclasts and osteoblasts is maintained. Our results demonstrate that RXR loss of function by targeted deletion in bone marrow hematopoietic progenitors impairs the acquisition of an active osteoclast phenotype during osteoclast differentiation without affecting osteoblast activity in vivo. Osteoclasts derived from RXR-deficient progenitors are abnormally large, which one would expect to be associated with high lytic activity (33). However, these osteoclasts have defects in bone resorption, which can be explained by their deficient expression of osteoclast activity genes and failure to form cytoskeletal structures necessary for resorption of the bone matrix. This eventually results in increased bone mass in male mice and protection from bone loss in an experimental model of postmenopausal osteoporosis. Our findings represent the first demonstration, to our knowledge, of the existence of an RXR-signaling pathway in osteoclastogenesis.

The skeletal phenotype of the RXR-KO male mice develops at the age when bone mass peaks (34) and osteoclast activity exceeds osteoblast activity (35). Under these conditions, the resorption deficiency of the RXR-KO osteoclasts may favor the 
A

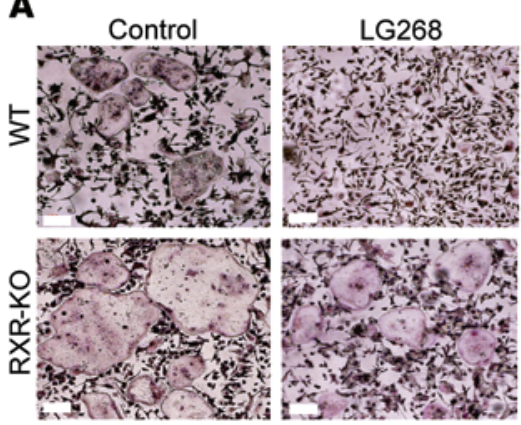

D

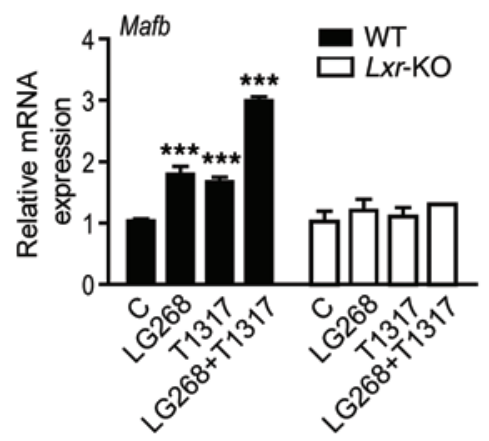

B

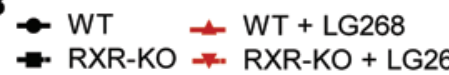

드. ${ }^{M a f b}$

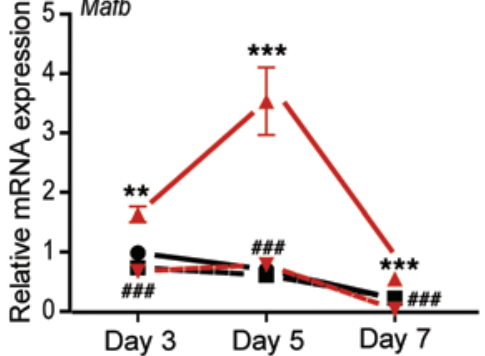

E

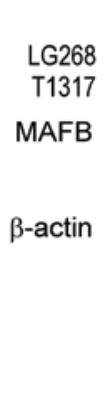

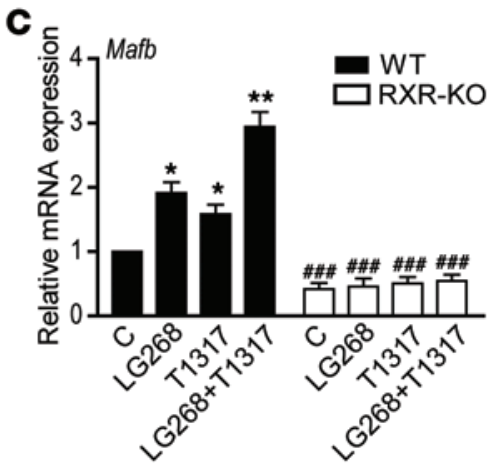

$\mathbf{F}$

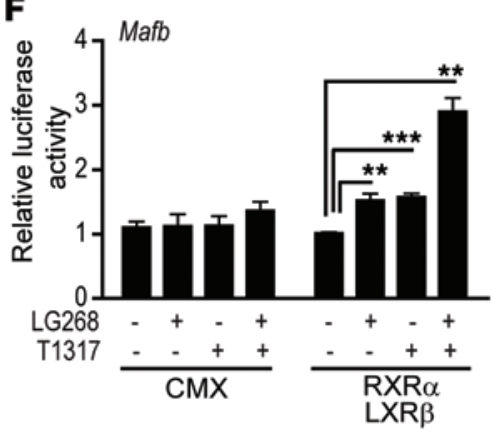

Figure 7. Pharmacological activation of RXR/LXR heterodimers blocks osteoclast differentiation through upregulation of MAFB. (A and B) In vitro osteoclast differentiation from WT and RXR-KO bone marrow cells, treated with vehicle (control) or LG268. (A) Representative mature osteoclasts identified as multinucleated TRAP ${ }^{+}$cells. Scale bars: $100 \mu \mathrm{m}$. (B) Mafb mRNA expression over a time course of osteoclast differentiation. ${ }^{* *} P<0.01$; ${ }^{* *} P<0.001$, compared with WT. $\# \# P<0.001$ for RXR-KO + LG268 compared with WT + LG268. (C and D) Mafb mRNA expression in WT and RXR-KO or Lxr-KO osteoclast progenitors treated with vehicle (C) or ligands for RXR (LG268) and LXR (T1317). ${ }^{*} P<0.05$; ${ }^{* *} P<0.01$; ${ }^{* * *} P<0.001$, compared with WT vehicle-treated cells (C) (paired 2-tailed Student's $t$ test), \#\# $P<0.001$ versus the equivalent treatment in WT cells (unpaired 2-tailed Student's $t$ test). (E) MAFB protein in WT and RXR-KO or $L x r-K O$ osteoclast progenitors treated with vehicle or ligands for RXR (LG268) and LXR (T1317); representative of 3 independent experiments. (F) Luciferase reporter assay in RAW264.7 cells transfected with RXR $\alpha$ and LXR $\beta$ or CMX empty vector together with a reporter vector containing $1.5 \mathrm{~kb}$ of the Mafb promoter. Data are presented relative to values obtained with the vehicle-treated Mafb reporter in the absence of RXR $\alpha$ and $\mathrm{LXR} \beta$. ${ }^{* *} P<0.01$; ${ }^{* *} P<0.001$. Data are presented as mean \pm SEM ( $n=3$ per group); statistical comparisons were made by paired ( $\mathbf{D}$ and $\mathbf{F}$ ) or unpaired (B) 2-tailed Student's $t$ test.

increase of bone mass. Interestingly, the basal skeletal phenotype of female mice was unaffected by RXR-deficient bone marrow hematopoietic progenitors. This might be explained by the effects of estrogens on osteoclastogenesis and osteoclast activity. Estrogens reduce osteoclast size $(36,37)$ and bone resorption $(37)$, and estrogens in female mice may thus impede the development of the bone phenotype seen in RXR-KO male mice. Supporting this idea, OvX-induced estrogen deficiency allowed female mice to develop the osteoclast RXR-dependent bone phenotype seen in male mice. Mutual interactions exist between RXRs and estrogen receptors that could influence their transcriptional activities, including direct protein-protein interactions (38), competition for DNA-binding domains (38), and competition for shared coactivators and corepressors (39). These interactions point to a potential mechanism through which estrogens could contribute to the transcriptional modulation of RXRs to affect osteoclast function in vivo. However, further studies are needed to assess the possibility of crosstalk between RXRs and estrogen receptors in bone.

Our investigation into the molecular mechanism identified Mafb as an RXR homodimer target in osteoclast progenitors under basal physiological conditions. MAFB has been shown to function as a corepressor of several osteoclastic transcription factors, and its expression is downregulated during osteoclas- togenesis (31). Despite this antiosteoclastogenic role, we found that MAFB expression in osteoclast progenitors is necessary for their proper proliferation and further differentiation into functional osteoclasts (Figure 10). A role for MAFB in the control of $\mathrm{M}-\mathrm{CSF}$-dependent proliferation of myeloid cells has been previously described $(26,27)$. Our studies demonstrate that MAFB controls M-CSF-dependent proliferation of osteoclast progenitors and that this has an impact on osteoclastogenesis. Indeed, osteoclasts differentiated from $M a f b$-silenced precursors are giant osteoclasts with low lytic activity, likely due to low expression of cathepsin-K, which encodes an important osteoclast protease whose deletion leads to osteopetrosis in mice (13). Although low cathepsin-K expression might account for the low lytic activity of RXR-KO osteoclasts, we cannot exclude the possibility that other genes regulating osteoclast activity might be direct targets of RXR and that this might influence the expression profile at later stages of osteoclast differentiation. For instance, regulation of Fos by RXR in a heterodimer with PPAR $\gamma(40)$ could contribute to the RXR-KO osteoclast phenotype.

Since their discovery, RXRs have been mainly studied as subordinate partners of other nuclear receptors (8). More recent studies have demonstrated that activation of RXRs regulates gene transcription (8). In addition, RXRs are known to form homodimers (8); 
A

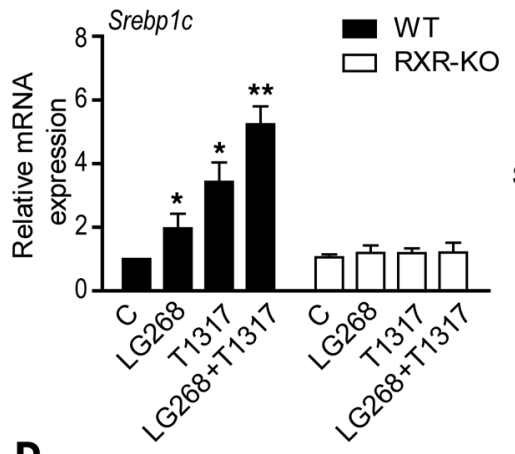

D

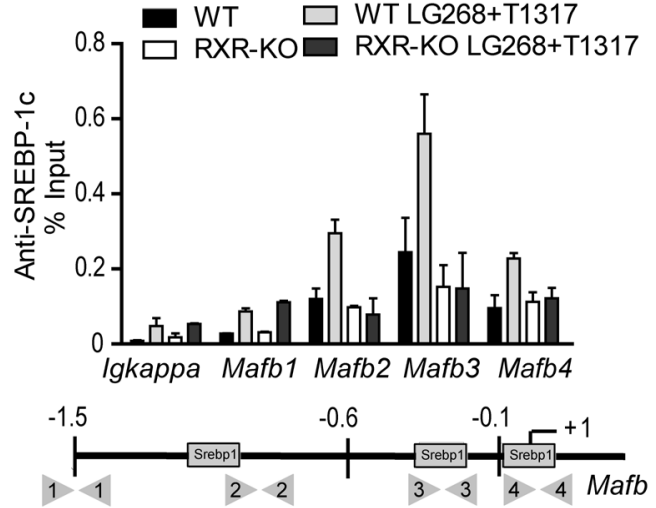

B

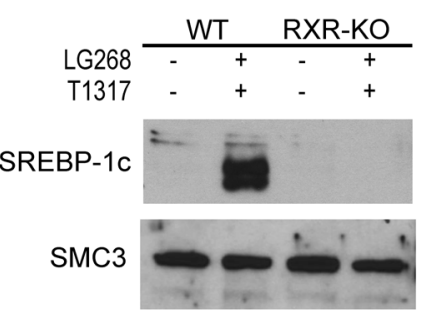

C

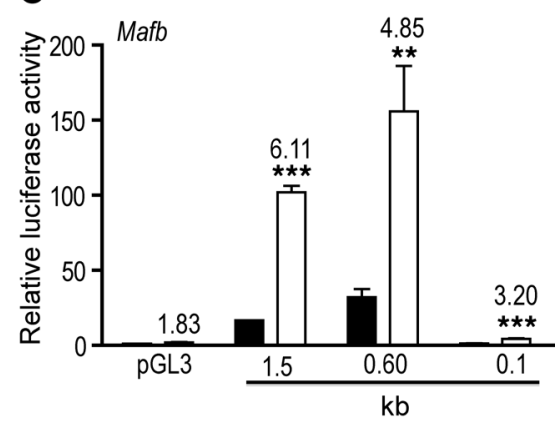

$\mathbf{F}$

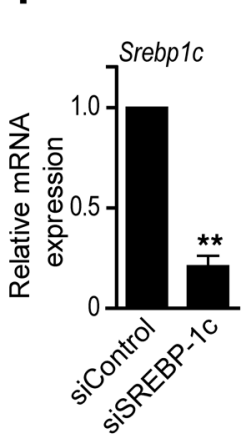

Figure 8. RXR/LXR induces Mafb transcription though SREBP-1c. (A) Srebp1c mRNA expression in WT and RXR-KO osteoclast progenitors treated with vehicle (C) or ligands for RXR (LG268) and LXR (T1317). ${ }^{*} P<0.01$; ${ }^{* *} P<0.001$, compared with WT vehicle-treated cells (C). (B) SREBP-1c protein expression in nuclear extracts from osteoclast progenitors treated with vehicle or ligands for RXR (LG268) and LXR (T1317); SMC3 was used as loading control. (C) Luciferase reporter assay in RAW264.7 cells transfected with SREBP-1c or empty cDNA3.1 vector together with the indicated Mafb promoter or pGL3 control reporters; data are presented relative to values obtained with the vehicle-treated pGL3 reporter in the absence of SREBP-1c (cDNA3.1). Indicated fold inductions represent (SREBP-1c)/(cDNA3.1). ${ }^{* *} P<0.01 ;{ }^{* *} P<0.001$, compared with reporter activity in the absence of SREBP-1c. (D) ChIP analysis of SREBP-1c binding to $-1.5 \mathrm{~kb}$ (Mafb1), $-1.0 \mathrm{~kb}$ (Mafb2), $-0.4 \mathrm{~kb}$ (Mafb3), and transcription starting (Mafb4) regions of the Mafb promoter or to a negative control (Igkappa) in osteoclast progenitors. Lower panel, localization of SREBP-1c-binding sites in the Mafb promoter. Arrows indicate the position of primers used for ChIP; the experiment shown is representative of 3 done in triplicate. mRNA expression of Mafb (E) and Srebp1c (F) in osteoclast progenitors transfected with control siRNA (siControl) or Srebp1c siRNA (siSREBP-1c). Data are presented as mean \pm SEM $\left(n=3\right.$ per group). ${ }^{*} P<0.01 ;{ }^{* *} P<0.001$, by paired (A) or unpaired (C, E, and $\mathbf{F}$ ) 2-tailed Student's $t$ tests.

however, very little is known about their in vivo functions $(8,29)$. Our analysis demonstrates that the role of RXR signaling in osteoclastogenesis under basal physiological conditions is independent of RXR heterodimeric partners with known roles in bone physiology (40-44). Among all the RXR heterodimeric partner-deficient mice, only PPAR $\gamma$ - $(40)$ and LXR-deficient mice $(42,43)$ increase bone mass, similar to our RXR-KO mice. However, a recent study showed that PPAR $\gamma$ deletion completely blocks osteoclast differentiation (40) and our in vitro studies and previous reports show that osteoclasts from LXR-deficient mice are morphologically indistinguishable from WT osteoclasts $(42,43)$. The osteoclast phenotype of mice deficient in PPAR $\gamma$ or LXR thus does not resemble the RXR$\mathrm{KO}$ osteoclast phenotype. In addition, steady-state levels of Mafb do not decrease in Pparg-KO and Lxr-KO osteoclast progenitors, indicating that the mechanisms leading to increased bone mass in Pparg-KO and Lxr-KO mice are different from the RXR homodimer-dependent mechanism described in the present study. Our results support an in vivo role for RXR homodimers, indicating that they can function as biologically relevant transcription units. It is notable that the regulation of $M a f b$ by RXR homodimers occurs in the absence of any exogenous RXR ligand, suggesting that RXR endogenous ligands might be produced during osteoclastogenesis. Supporting this idea, several fatty acids such as arachidonic acid and docosahexaenoic acid, which are ligands of RXR (45), have been shown to play a role in osteoclastogenesis $(46,47)$. Further studies are required to determine whether endogenous RXR ligands are physiologically relevant to the control of osteoclast differentiation and activation.

Unexpectedly, given the effect of RXR deficiency under basal conditions, we found that pharmacological activation of RXR inhibits osteoclastogenesis. This effect is likely due to MAFB-mediated blockade of RANKL signaling in differentiating osteoclasts (31), since RXR agonists upregulate MAFB expression during the course of osteoclast differentiation (Figure 10). Our findings are in agreement with previous studies showing an inhibitory effect of retinoids on osteoclast differentiation $(48,49)$. All-trans retinoic acid, through activation of RAR, suppresses the downregulation of MAFB during osteoclast differentiation (48). However, in agree- 
A

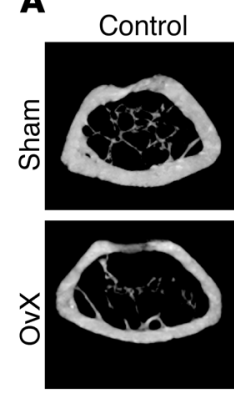

BXR

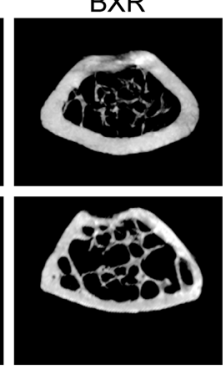

B

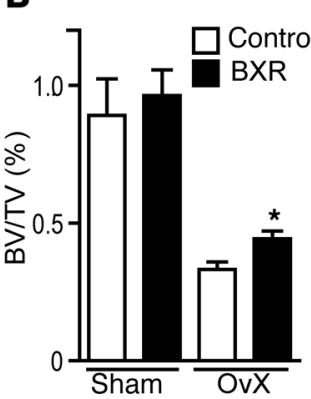

C

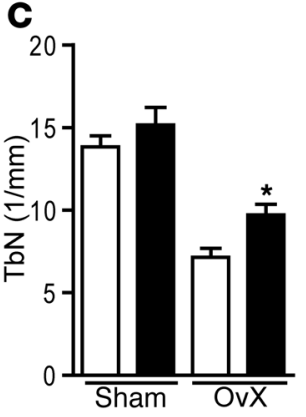

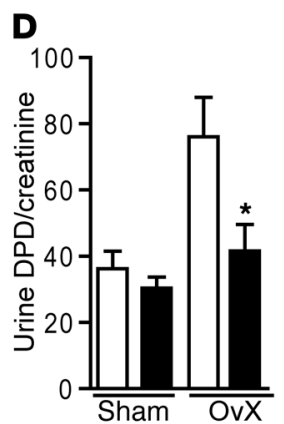

$\mathbf{E}$

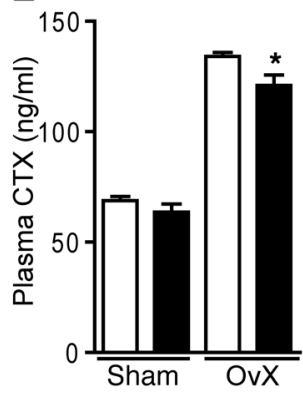

Figure 9. Protective effect of pharmacological RXR activation on bone loss. (A) Representative $\mu$ CT images of 3 mice per genotype, showing the femur of control and bexarotene-treated female mice that underwent sham surgery or OvX. BXR, bexarotene. (B and C) Histomorophometric analysis of the tibial end of the femur. $n=8$ per group. ( $\mathbf{D}$ and $\mathbf{E}$ ) Levels of plasma and urine osteoclast activity markers. $n=5$ in sham-treated and $n=6$ in OvX per group. 20-week-old female mice were used for these experiments; data are presented as mean \pm SEM. ${ }^{*} P<0.05$, compared with vehicle-treated ovariectomized mice (unpaired 2-tailed Student's $t$ test).

ment with another study (49), we did not detect any effect of the RAR-selective ligand TTNPB on the expression of Mafb. We demonstrate here that the inhibition of osteoclastogenesis mediated by the selective pharmacological activation of RXR does not occur through its heterodimerization with RAR, but with LXR. Our results indicate that upon RXR activation, RXR/LXR indirectly regulates $M a f b$ expression through the induction of SREBP-1c, a master regulator of lipid homeostasis (50). Our findings suggest that the control of Mafb expression by SREBP-1c might be a link between lipid and bone metabolism $(51,52)$. Interestingly, treatment with statins, a class of drugs used to lower cholesterol levels, reduces the number of differentiated osteoclasts and the risk of bone fracture (53). In addition, the finding that SREBP-1 directly regulates the expression of $M a f b$ in osteoclasts raises the possibility that SREBP-1 may influence cell functions beyond their role as regulators of lipid metabolism. Further studies are needed to explore the putative role of SREBP-1 in osteoclastogenesis.

The effect of pharmacological RXR activation on osteoclast differentiation occurs through heterodimerization with LXR, but not with other nuclear receptors expressed in osteoclast progenitors, such as PPAR $\gamma$ (30). This finding is particularly relevant, since PPAR $\gamma$ ligands have negative effects on bone anabolism in mice and humans (30), which challenges their therapeutic benefits as insulin sensitizers. Our results demonstrate that, in contrast with PPAR $\gamma$ activation, RXR activation has no negative effects on physiological bone turnover and moreover show that treatment with bexarotene reduces pathological osteoclast activation and bone resorption in a mouse model of postmenopausal osteoporosis. These results suggest that RXR homodimer inhibition in bone marrow progenitors or activation of RXR in differentiating osteoclasts may be beneficial for the treatment of bone diseases associated with increased osteoclast activity, such as osteoporosis. In addition, since RXR ligands are also insulin sensitizers (54), our studies suggest that RXR-specific modulators (8) might provide an alternative to PPAR $\gamma$ agonist antidiabetic drugs without compromising bone homeostasis.

\section{Methods}

Mice. In order to conditionally ablate the RXR isoforms expressed by hematopoietic cells ( $R X R \alpha$ and $R X R \beta)$ we used the Mx1-Cre transgenic mouse system on the C57BL/6 background. We crossed mice expressing the bacteriophage P1-derived Cre/loxP recombinase under the control of the Mx promoter with mice bearing loxPflanked $R x r a^{f / f l}$ and $R x r b^{f / f l}$ sequences. Mice carrying $R x r a^{f / f l}$ and $R x r b^{A / f l}$ have been described elsewhere $(11,55) . R x r b^{f / f l}$ mice were donated by Pierre Chambon (Université de Strasbourg). RXR deletion was induced by injecting pI:pC at days 3, 5, and 7 after birth. Mice were genotyped by PCR using primers provided in Supplemental Table 1: P1, P2, and P3 for RXR $\alpha$ (11); and XO141 and WS55 for

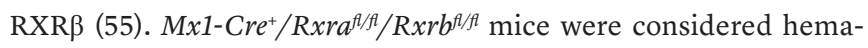
topoietic cell-specific KOs for RXR $\alpha$ and RXR $\beta$ (RXR-KO). Their

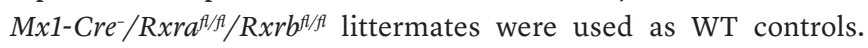
WT and RXR-KO male mice were studied at 8 and 20 weeks of age. WT and RXR-KO female mice were studied at 20 weeks of age. $\mathrm{M} x 1-\mathrm{Cr}^{+} /{\mathrm{R} x r a^{f / f}}^{\prime / f}$ mice have been previously described (11). LXRdeficient mice $(56,57)$ were donated by David Mangelsdorf (University of Texas Southwestern, Dallas, Texas, USA). Pparg-KO mice have been described previously (58). Mice were fed with normal chow diet (ssniff). In order to test the effects of RXR activation on bone homeostasis, 8-week-old male C57BL/6 mice (Charles River) were orally gavaged daily for 6 weeks with $35 \mathrm{mg} / \mathrm{kg} / \mathrm{d}$ bexarotene (LGD1069) or vehicle.

OvX. For OvX experiments, mice were bilaterally ovariectomized, and the ovaries of the sham-operated group were left intact. Both sets of mice were kept in identical housing conditions controlling for the same environment, food, light, and temperature conditions. In order to test the effects of RXR deletion on bone homeostasis, we used WT and RXR-KO female mice at 20 weeks of age. Mice were killed 8 weeks after OvX for further analysis. To assess the effects of RXR activation on bone loss, 20-week-old C57BL/6 mice were ovariectomized, and thereafter the mice were orally gavaged with $35 \mathrm{mg} / \mathrm{kg} / \mathrm{d}$ of bexarotene (LGD1069) or vehicle on a daily basis. For analysis, mice were killed after 8 weeks of treatment.

Ligands. GW327647 (GW647) and GW610742X (GW742) were provided by Tim Willson (GlaxoSmithKline). LG268 and LG754 were a gift from Reid Bissonette (Ligand Pharmaceutical). Rosiglitazone (Rosi) and T1317 were purchased from Cayman Chemical, GW3965 from Tocris Bioscience, and 9cRA, TTNPB, 1 $\alpha, 25$-dihydroxyvitamin D3 (VitD), and triidothyronine (T3) from Sigma-Aldrich. Bexarotene (\#17743851) was purchased from Molekula. 

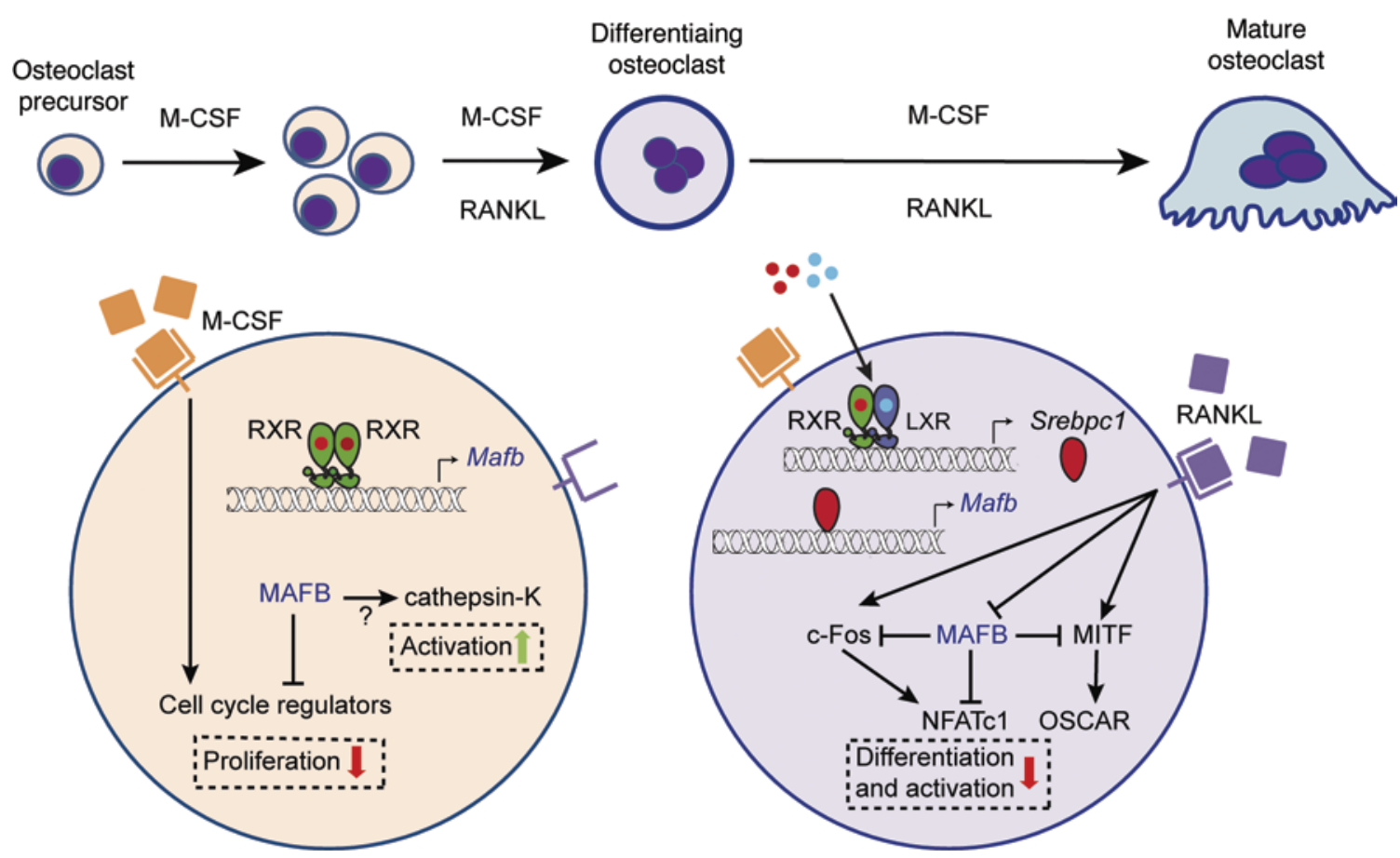

Figure 10. Schematic of RXR/MAFB signaling in osteoclastogenesis. RXR homodimers sustain Mafb transcription in osteoclast precursors, allowing a proper proliferative response to M-CSF and expression of cathepsin-K during osteoclast differentiation. In differentiating osteoclasts, ligand activation of RXR/LXR heterodimers induces the expression of Mafb through SREBP-1c. As a result, MAFB counteracts RANKL signaling and inhibits osteoclast differentiation and activation.

Assessment of bone homeostasis. Bone parameters were assessed in male mice at 8 and 20 weeks of age and in female mice at 20 weeks of age. In female mice, we also assessed these parameters 8 weeks after OvX or sham surgery. To characterize bone architecture, samples were fixed in $4 \%$ paraformaldehyde (PFA) diluted in PBS (0.1 M, pH 7.4) for 72 hours. Tissues were decalcified in $10 \%$ EDTA in PBS at $37^{\circ} \mathrm{C}$ for 48 hours, dehydrated, cleared in xylene, and finally embedded in Paraplast embedding medium. Microtome sections $(5 \mu \mathrm{m})$ were cut and stained with Harris H\&E (Sigma-Aldrich). Histomorphometric analysis was carried out on digital images taken at $\times 200$ magnification (59) using Imaris and Nikon NIS Elements image analysis software. Osteoclasts were labeled for TRAP enzyme activity with the leukocyte acidic phosphatase kit (Sigma-Aldrich). Osteoblasts were visualized by alkaline phosphatase (ALP) histochemistry (Sigma-Aldrich). Mineral apposition rate was determined with double-calcein labeling (60). For analysis of osteoclast ultrastructure, samples were cut from the epiphyseal region of the femur and immersed immediately in a mixture of PFA and glutaraldehyde (4/1). Samples were processed for TEM as described (61).

In vivo markers of bone turnover. To determine osteoclast and osteoblast activity, we assayed plasma TRAP, ALP, OCN, CTX, and the urine DPD/creatinine ratio using commercial ELISA kits (TRAP and CTX: Immunodiagnostic Systems; DPD: Bender MedSystems; OCN: BTI Biomedical Technologies; ALP: Alpha Diagnostics; creatinine: Spinreact). Plasma samples were obtained by venipuncture, and urine samples were pooled for 24 hours in individual urine chambers (Tecniplast).

DEXA and ex vivo high-resolution $\mu C T$. BMD and BMC were measured by DEXA (Lunar PIXImus) (40). Femurs were isolated and air dried, and $\mu \mathrm{CT}$ scans were then acquired with the use of a Skyscan-1072 high resolution in vitro $\mathrm{x}$-ray microtomograph.
In vitro osteoclast differentiation. Total bone marrow cells from 6to 8-week-old mice were differentiated in vitro to mature osteoclasts in 24-well or 96-well plates. Cells were cultured in $\alpha$-MEM containing $10 \% \mathrm{FBS}, 60 \mathrm{ng} / \mathrm{ml}$ of recombinant murine ( $\mathrm{rm}$ ) M-CSF (PeproTech), and 15 or $50 \mathrm{ng} / \mathrm{ml}$ of rm-RANKL (R\&D Systems) for 24-well or 96-well plates, respectively. For these experiments, male and female mice were used. After 5 to 7 days of treatment, cultured cells were fixed and stained for TRAP, and TRAP-positive multinucleated cells $(n>3)$ were counted. Nuclei number per cell was determined after DAPI staining, and the osteoclast area was analyzed using Sigma ScanPro Image software. Osteoclast progenitors were obtained from total bone marrow cells after 3 days of culture in medium containing M-CSF (30 ng/ml).

Measurement of osteoclast lytic activity. To determine osteoclast lytic activity, osteoclast bone resorption capacity was assayed by differentiating osteoclasts as described above, using calcium-phosphatecoated 24-well resorption assay plates (Cosmo Bio Co.) or 96-well plates containing bovine cortical bone slices (DT-1BON1000-96, Immunodiagnostic Systems Ltd). At day 7 of osteoclast differentiation, cells were removed and the remaining calcium-phosphate matrix of the plate and the resorption pits formed on the bone slices were stained with $0.01 \%$ Toluidine Blue (Sigma-Aldrich). The resorption pit area was determined with Sigma ScanPro image analysis software by counting 100 resorption pits in each sample.

Real-time imaging of osteoclast motility. Osteoclasts were cultured in glass bottom culture dishes (MatTek Co.) and subsequently analyzed with a Leica DIC microscope. Image sequences were reconstructed using ImageJ software (http://imagej.nih.gov/ij/).

Labeling of osteoclast cytoskeleton. For visualization of the osteoclast cytoskeleton, total bone marrow cells were seeded on glass cov- 
erslips or bovine cortical bone slices (donated by T. Arnett, University College London, London, United Kingdom) and differentiated into osteoclasts as described above. On days 5 to 7, cells were fixed for 20 minutes in $4 \%$ PFA in PBS. Cells were washed 3 times with PBS, permeabilized with $0.5 \%$ Triton X-100 in PBS for 10 minutes, and blocked with $5 \%$ normal goat serum for 45 minutes at room temperature. Actin filaments were detected by incubation with a solution of $0.1 \mu \mathrm{g} / \mathrm{ml}$ Alexa Fluor 488-conjugated phalloidin (Molecular Probes) in PBS for 1 hour. For localization of WASP, cells were incubated for 1 hour at room temperature with a $1 / 500$ dilution of anti-WASP antibody (\#8353, Santa Cruz Biotechnology Inc.) in PBS containing 2\% BSA, followed by incubation with Alexa Fluor 488-conjugated anti-rabbit IgG. Actin rings were analyzed with a Zeiss LSM confocal microscope, which allowed us to identify distinct podosome arrangements: (a) complete podosome belt, with a belt-like arrangement of podosomes at the cell periphery: individual podosomes cannot be distinguished; (b) incomplete podosome belt, with circular clusters of podosomes and scattered podosomes behind the cell leading edge; (c) complete actin ring, with podosomes arranged in a ring-like structure at the cell periphery: individual podosomes cannot be distinguished; and (d) incomplete actin ring, with abundant podosome clusters behind the cell leading edge: individual podosomes can be distinguished. We counted 100 cells per experimental condition, analyzing cells in at least 4 distinct fields of each sample, and quantification was blinded to the genotypes.

Immunophenotyping and cell sorting. Cell suspensions were prepared from freshly harvested femur bone marrow. For immunophenotyping, cells were analyzed with a BD FACSCanto Flow Cytometer and BD FACSDiva software (BD Biosciences). We detected hematopoietic and progenitor cell subpopulations using the following antibodies: biotin-conjugated lineage panel antibodies (anti-CD3e, antiCD11b, anti-CD45R/B220, anti-GR-1, and anti-Ter119; \#559971, BD Biosciences - Pharmingen), Pe.Cy7-labeled anti-Sca-1 (\#558162, BD Biosciences - Pharmingen), APC-labeled anti-c-Kit (\#553356, BD Biosciences - Pharmingen), EF700-labeled anti-CD16/32 (\#560161-80, eBioscience), and FITC-labeled anti-CD34 (\#560238, BD Biosciences - Pharmingen). For the detection of mature myeloid subpopulations, the following antibodies were used: Pacific Blue-labeled anti-CD45 (\#48-0451-82, eBioscience), PeCy7-labeled anti-Cd11b (\#552850, BD Biosciences - Pharmingen), PE-labeled anti-Ly6G (\#551461, BD Biosciences - Pharmingen), and FITC-labeled Ly6C (\#553104, BD Biosciences - Pharmingen). For the isolation of myeloid subpopulations, cells were sorted according to CD31 and Ly6C expression, as previously described (62). We used the MoFlo (Modular Flow) High-Performance Cell Sorter (Dako) and the following antibodies: APC-labeled anti-CD31 (\#551262, BD Biosciences - Pharmingen) and FITC-labeled anti-Ly6C (\#553104, BD Biosciences - Pharmingen). Lineage $^{-}\left(\right.$lin $\left.^{-}\right)$and Lineage ${ }^{+}\left(\right.$lin $\left.^{+}\right)$bone marrow fractions were isolated with an indirect magnetic labeling system (Miltenyi Biotec).

Gene expression analysis. For quantitative PCR (qPCR) analysis in osteoclast progenitors, cells were treated for 6 hours with ligands of RXR or its heterodimeric partners. We used the RXR ligands LG268 (100 nM), 9cRA (100 nM), and LG754 (1 $\mu \mathrm{M})$. Cells were treated with GW3965 $(1 \mu \mathrm{M})$ and T1317 $(1 \mu \mathrm{M})$ for LXR activation; XCT0135908 $(1 \mu \mathrm{M})$ for Nurr1; GW647 (100 nM) for PPAR $\alpha$; GW742 (100 nM) for $\operatorname{PPAR} \delta / \beta$; and Rosi $(1 \mu \mathrm{M})$ for PPAR $\gamma$. We used VitD (40 nM) for VDR activation; T3 (50 nM) for TR; and TTNPB $(1 \mu \mathrm{M})$ for RAR. For qPCR analysis during osteoclast differentiation in vitro, cells were treated with LG268 $(1 \mu \mathrm{M})$ or T1317 $(1 \mu \mathrm{M})$. Total RNA from bone, osteoclast progenitors, or mature osteoclasts was isolated using TrizoL (SigmaAldrich) or RNeasy Mini Kit (QIAGEN). Transcripts were quantified in a 2-step reverse-transcription qPCR process (61). Gene expression values were normalized to the housekeeping genes $36 b 4$ and cyclophilin and expressed as relative mRNA levels or fold changes compared with untreated controls. Data were analyzed using qBASE (Biogazelle). Primer sequences are provided in Supplemental Table 1.

Cell extract preparation and Western blot analysis. We used osteoclast progenitors or total bone marrow for Western blotting. When necessary, cells were treated for 24 hours with LG268 (100 nM), 9cRA $(100 \mathrm{nM})$, or T1317 $(1 \mu \mathrm{M})$. For preparation of total lysates, cells were washed in PBS and incubated for 20 minutes at $4^{\circ} \mathrm{C}$ in RIPA lysis buffer (50 mM Tris- $\mathrm{HCl}, \mathrm{pH}$ 8.0, $150 \mathrm{mM} \mathrm{NaCl}, 0.1 \%$ SDS, $1 \% \mathrm{NP}-40$, $0.5 \% \mathrm{C}_{24} \mathrm{H}_{39} \mathrm{NaO}_{4}$ and protease inhibitors; Sigma-Aldrich). Lysates were centrifuged $(14,000 \mathrm{~g}, 15$ minutes), the protein concentration in the supernatants was measured (Bio-Rad), and solubilized proteins $(40 \mu \mathrm{g})$ were separated by $10 \%$ SDS-PAGE. For SREBP-1 immunoblotting, we used purified nuclei prepared from osteoclast progenitors as described before (63). After transfer, blocked membranes were probed with the following antibodies: RXR $\alpha$ (D-20, \#sc-553, Santa Cruz Biotechnology Inc.), RXR $\beta$ (S-20, \#sc-554, Santa Cruz Biotechnology Inc.), MAFB (\#ab66506, Abcam), or SREBP-1 (\#MS-1207, Thermo Scientific). For loading control of total and nuclear extracts, we used antibodies against $\beta$-actin (\#A2066, Sigma-Aldrich) and SMC3 (\#AB3914, EMD Millipore), respectively.

Proliferation assays. Freshly isolated bone marrow cells from 6- to 10-week-old male and female mice were incubated for 12 days in Methocult-3234 (Stem Cell Technologies) supplemented with $100 \mathrm{ng} / \mathrm{ml}$ M-CSF (PeproTech). CFUs were determined. For cell division analysis, osteoclast progenitors were stained with violet proliferation dye 450 (V450, BD Horizon) and incubated overnight in RPMI containing $0.5 \%$ FBS. Cells were then incubated in RPMI with 10\% FBS and $50 \mathrm{ng} / \mathrm{ml} \mathrm{M-CSF}$ for 12, 24, 36, or 48 hours. The percentage of osteoclast progenitors that had undergone mitosis (\% proliferation) was determined by the V450 dilution assay using a BD FACSCanto flow cytometer (BD Biosciences) and subsequent FlowJo data analysis.

Lentiviral gene transduction. The mouse Mafb cDNA (a gift from M. Sakai, University of Hokkaido, Hokkaido, Japan) was amplified and cloned into pHRSIN CSGW-dlNotI lentiviral vector (provided by M.K. Collins, University College London), together with the cDNA for IRES GFP. The primers used for amplification are provided in Supplemental Table 1. 293T cells (obtained from ATCC) were used to produce viral particles using the 3 plasmid HIV-derived lentiviral system (provided by M.K. Collins, University College London) and the pMD2.G vector (\#12259, Addgene Plasmid). Control viral particles were also generated using a vector encoding GFP alone. For viral inoculation, we incubated total bone marrow cells or osteoclast progenitors with the viral supernatants $(\mathrm{MOI}=3$ ) in $\alpha$-MEM (supplemented with $10 \% \mathrm{FBS}$, $\beta$-mercaptoethanol, and $40 \mathrm{ng} / \mathrm{ml} \mathrm{M}$-CSF) for 48 hours at $37^{\circ} \mathrm{C}$. We then removed the viral supernatants and performed CFU and proliferation assays as described above. GFP and MAFB expression signals in infected osteoclast progenitors were detected by fluorescent microscopy and Western blotting, respectively.

Promoter analyses. For in silico evaluation of the Mafb promoter, we used "Mulan" (64), and HOMER and Motif Matcher from UCSC (http://genome.ucsc.edu) (65). For transient transfection, we used 
Lipofectamine 2000 (Invitrogen) as previously described (66). We transfected Mafb luciferase reporters (donated by M. Sakai, University of Hokkaido) or the pGL3 control reporter into the mouse macrophage cell line RAW264.7 (obtained from ATCC) together with expression plasmids for RXR $\alpha$, LXR $\beta$, or SREBP-1c; pCMX or pcDNA3 empty vectors were used as negative controls. When necessary, we treated the cells the next day with $1 \mu \mathrm{M}$ LG268, 9cRA, LG754, and T1317, or ethanol vehicle, for 18 hours. Results are expressed as relative promoter activity levels (fold change) compared with untreated controls. For ChIP analysis of RXRs, osteoclast progenitors were treated for $15 \mathrm{~min}$ utes with LG268 $(1 \mu \mathrm{M})$ or 9cRA $(1 \mu \mathrm{M})$; for ChiP analysis of SREBP-1c, cells were treated for 4 hours with LG268 $(1 \mu \mathrm{M})$ and T1317 $(1 \mu \mathrm{M})$. In both cases, ChIP assays were performed as previously described (67). For the detection of RXR and SREBP-1c binding to the Mafb promoter, we used a combination of 2 RXR-specific antibodies (D-20, \#sc-553 and $\Delta \mathrm{N}$ 197, \#sc-774, Santa Cruz Biotechnology Inc.) or an SREBP-1cspecific antibody (H-160, \#sc-8984, Santa Cruz Biotechnology Inc.). ChIP output was measured by qPCR and normalized to the $1 \%$ input. Primer sequences are provided in Supplemental Table 1. ChIP-seq data were deposited in the NCBI's Gene Expression Omnibus database (GSE63698 [RXR, PPAR $\gamma$, and SREBP-1]; GSE21512 [LXR]).

Mafb and Srebp1c silencing. We used predesigned mouse Mafb siRNA (\#ID 156036, Ambion) and Srebp1c siRNA (\#NM_011480si.1, Eurofins, MWG Operon). Control siRNA was purchased from Dharmacon. For Mafb silencing, osteoclast progenitors were differentiated in 24-well plates as described above. At day 2 of differentiation, cells were transfected using Lipofectamine RNAiMAX reagent (Invitrogen) in the presence of M-CSF (60 ng/ml) and RANKL (15 ng/ml). After 4 hours, the medium was removed and osteoclast differentiation was continued for another 3 days. For TRAP staining and RNA isolation, cells were processed as described above. For measurement of lytic activity, osteoclasts were scraped from the wells into suspension as described previously (68), and $100 \times 10^{3}$ mature osteoclasts were plated on bone slices in 96-well plates and cultured in the presence of M-CSF $(60 \mathrm{ng} / \mathrm{ml})$ and RANKL ( $50 \mathrm{ng} / \mathrm{ml}$ ) for an additional 2 days. Bone resorption was measured as described above. For Srebp1c silencing, siRNAs were transfected into osteoclast progenitors using Lipofectamine 2000 (Invitrogen). Subsequent assays were carried out after 12 hours of transfection. The effectiveness of the siRNAs used in these studies was checked by qPCR.
Statistics. Power analysis was performed (GPower 3.1) to determine study group size using preliminary data sets. Statistical significance was determined using a nonparametric, 2-tailed Mann-Whitney $U$ test for data with nonnormal distribution. Parametric 2-tailed Student's $t$ test was used for data with normal distribution. Results are shown as mean \pm SEM. Differences were considered significant at $P<0.05$. Statistical analysis was conducted with Prism version 5 (GraphPad Software).

Study approval. All experimental procedures were approved by the Experimental Animal Use Committee of the Instituto de Salud Carlos III and were in accordance with the EU Directive 86/609.

\section{Acknowledgments}

We thank F. Lakiere (University of Antwerp), C. Aguado (Universidad Autónoma, Madrid, Spain), G. Herrero-Beaumont and R. Largo (Fundación Jiménez Díaz, Madrid, Spain), S. Schmidt and P. Liu (Universität Ulm), and J.M. Fernández-Toro (Transgenesis Unit, CNIC) for technical help. We also thank N. Spann and C. Glass (UCSD, La Jolla, California, USA) and D. Alameda (CNIC) for access to ChIP-seq data; D. Mangelsdorf (University of Texas Southwestern) for LXR-deficient mice; P. Chambon (Université de Strasbourg) for Rxr $b^{A / f}$ mice; M. Sakai (University of Hokkaido) for MAFB plasmids; T. Arnett (University College London) for providing the bovine bone slices; L. Bakiri (CNIO, Madrid, Spain) and Y. Calle (University of Roehampton, London, United Kingdom) for critical review of the manuscript; and S. Bartlett (CNIC) for editorial assistance. This work was supported by grants from the Spanish Ministry of Economy and Competitiveness (SAF2012-31483 to M. Ricote; SAF2011-23402 to A.F. Valledor), a Juan de la Cierva Program and an FP7 Marie Curie Career Integration Grant (to L. Fuentes), and an FP7 Marie Curie IEF Grant and a European Study for Diabetes-Lilly Research Program grant (to T. Röszer). The CNIC is supported by the Spanish Ministry of Economy and Competitiveness and the Pro-CNIC Foundation.

Address correspondence to: Mercedes Ricote, Department of Cardiovascular Development and Repair, Fundación Centro Nacional de Investigaciones Cardiovasculares Carlos III, Calle Melchor Fernández Almagro 3, 28029 Madrid, Spain. Phone: 34.91.45312.00, ext.3306; E-mail: mricote@cnic.es.
1. Kanis JA, McCloskey EV, Johansson H, Oden A. Approaches to the targeting of treatment for osteoporosis. Nat Rev Rheumatol. 2009;5(8):425-431.

2. DiGirolamo DJ, Clemens TL, Kousteni S. The skeleton as an endocrine organ. Nat Rev Rheumatol. 2012;8(11):674-683.

3. O'Brien CA, Nakashima T, Takayanagi H. Osteocyte control of osteoclastogenesis. Bone. 2013;54(2):258-263.

4. Harvey N, Dennison E, Cooper C. Osteoporosis: impact on health and economics. Nat Rev Rheumatol. 2010;6(2):99-105.

5. Vanderschueren D, Boonen S, Bouillon R. Physiology of calcium homeostasis and bone remodeling. In: Geusens P, Sambrook P, Lindsay R, eds. Osteoporosis in Clinical Practice: A Practical Guide for Diagnosis and Treatment. 2nd ed. London, United Kingdom: Springer London; 2004:9-14.

6. Harre U, Schett G. Bone research in 2012: The ups and downs of bone in health and rheumatic disease. Nat Rev Rheumatol. 2013;9(2):67-68.

7. Rachner TD, Khosla S, Hofbauer LC. Osteoporosis: now and the future. Lancet. 2011;377(9773):1276-1287.

8. Rőszer T, Menéndez-Gutiérrez MP, Cedenilla M, Ricote M. Retinoid X receptors in macrophage biology. Trends Endocrinol Metab. 2013;24(9):460-468.

9. Miyazaki S, et al. Nuclear hormone retinoid X receptor (RXR) negatively regulates the glucosestimulated insulin secretion of pancreatic sscells. Diabetes. 2010;59(11):2854-2861.

10. Hamann C, Kirschner S, Gunther KP, Hofbauer LC. Bone, sweet bone - osteoporotic fractures in diabetes mellitus. Nat Rev Endocrinol. 2012;8(5):297-305.

11. Ricote M, Snyder CS, Leung HY, Chen J, Chien $\mathrm{KR}$, Glass CK. Normal hematopoiesis after con- ditional targeting of RXRalpha in murine hematopoietic stem/progenitor cells. J Leukoc Biol. 2006;80(4):850-861.

12. Kastner $P$, et al. Abnormal spermatogenesis in RXR beta mutant mice. Genes Dev. 1996;10(1):80-92.

13. Lotinun S, et al. Osteoclast-specific cathepsin K deletion stimulates S1P-dependent bone formation. J Clin Invest. 2013;123(2):666-681.

14. Tran Van P, Vignery A, Baron R. An electronmicroscopic study of the bone-remodeling sequence in the rat. Cell Tissue Res. 1982;225(2):283-292.

15. Lomaga MA, et al. TRAF6 deficiency results in osteopetrosis and defective interleukin-1, CD40, and LPS signaling. Genes Dev. 1999;13(8):1015-1024.

16. Kikuta J, Ishii M. Osteoclast migration, differentiation and function: novel therapeutic 
targets for rheumatic diseases. Rheumatology. 2013;52(2):226-234.

17. Bozec A, et al. Osteoclast size is controlled by Fra-2 through LIF/LIF-receptor signalling and hypoxia. Nature. 2008;454(7201):221-225.

18. Lees RL, Heersche JN. Macrophage colony stimulating factor increases bone resorption in dispersed osteoclast cultures by increasing osteoclast size. J Bone Miner Res. 1999;14(6):937-945.

19. Teitelbaum SL, Ross FP. Genetic regulation of osteoclast development and function. Nat Rev Genet. 2003;4(8):638-649.

20. Insogna KL, et al. Colony-stimulating factor-1 induces cytoskeletal reorganization and c-srcdependent tyrosine phosphorylation of selected cellular proteins in rodent osteoclasts. JClin Invest. 1997;100(10):2476-2485.

21. Calle $\mathrm{Y}$, et al. WASp deficiency in mice results in failure to form osteoclast sealing zones and defects in bone resorption. Blood. 2004;103(9):3552-3561.

22. Chiusaroli R, et al. Tyrosine phosphatase epsilon is a positive regulator of osteoclast function in vitro and in vivo. Mol Biol Cell. 2004;15(1):234-244.

23. Jurdic P, Saltel F, Chabadel A, Destaing O. Podosome and sealing zone: specificity of the osteoclast model. Eur JCell Biol. 2006;85(3):195-202.

24. Boyle WJ, Simonet WS, Lacey DL. Osteoclast differentiation and activation. Nature. 2003;423(6937):337-342.

25. de Vries TJ, Schoenmaker T, Hooibrink B, Leenen PJ, Everts V. Myeloid blasts are the mouse bone marrow cells prone to differentiate into osteoclasts. J Leukoc Biol. 2009;85(6):919-927.

26. Sarrazin S, et al. MafB restricts M-CSF-dependent myeloid commitment divisions of hematopoietic stem cells. Cell. 2009;138(2):300-313.

27. Aziz A, Soucie E, Sarrazin S, Sieweke MH. $\mathrm{MafB} / \mathrm{c}-\mathrm{Maf}$ deficiency enables self-renewal of differentiated functional macrophages. Science. 2009;326(5954):867-871

28. Lala DS, et al. Activation of specific RXR heterodimers by an antagonist of RXR homodimers. Nature. 1996;383(6599):450-453.

29. Nunez V, et al. Retinoid X receptor alpha controls innate inflammatory responses through the upregulation of chemokine expression. Proc Natl Acad Sci U S A. 2010;107(23):10626-10631.

30. Lefebvre P, Benomar Y, Staels B. Retinoid X receptors: common heterodimerization partners with distinct functions. Trends Endocrinol Metab. 2010;21(11):676-683.

31. Kim K, et al. MafB negatively regulates RANKLmediated osteoclast differentiation. Blood. 2007;109(8):3253-3259.

32. Teti A. Mechanisms of osteoclast-dependent bone formation. Bonekey Rep. 2013;2:449.

33. Fujita $\mathrm{K}$, et al. Vitamin $\mathrm{E}$ decreases bone mass by stimulating osteoclast fusion. Nat Med. 2012;18(4):589-594.

34. Jilka RL. The relevance of mouse models for investigating age-related bone loss in humans. J Gerontol A Biol Sci Med Sci. 2013;68(10):1209-1217.

35. Cao J, Venton L, Sakata T, Halloran BP. Expression of RANKL and OPG correlates with agerelated bone loss in male C57BL/6 mice. J Bone Miner Res. 2003;18(2):270-277.

36. Liu CC, Howard GA. Bone-cell changes in estrogen-induced bone-mass increase in mice: dissociation of osteoclasts from bone surfaces. Anat Rec. 1991;229(2):240-250.

37. Takano-Yamamoto T, Rodan GA. Direct effects of 17 beta-estradiol on trabecular bone in ovariectomized rats. Proc Natl Acad SciU S A. 1990;87(6):2172-2176.

38. Song MR, Lee SK, Seo YW, Choi HS, Lee JW, Lee MO. Differential modulation of transcriptional activity of oestrogen receptors by direct proteinprotein interactions with retinoid receptors. Biochem J. 1998;336(pt 3):711-717.

39. Ellmann S, Sticht H, Thiel F, Beckmann MW, Strick R, Strissel PL. Estrogen and progesterone receptors: from molecular structures to clinical targets. Cell Mol Life Sci. 2009;66(15):2405-2426.

40. Wan Y, Chong LW, Evans RM. PPAR- $\gamma$ regulates osteoclastogenesis in mice. Nat Med. 2007;13(12):1496-1503.

41. Bland R. Steroid hormone receptor expression and action in bone. Clin Sci (Lond). 2000;98(2):217-240.

42. Robertson KM, et al. Cholesterol-sensing receptors, liver X receptor alpha and beta, have novel and distinct roles in osteoclast differentiation and activation. J Bone Miner Res. 2006;21(8):1276-1287.

43. Remen KM, Henning P, Lerner UH, Gustafsson JA, Andersson G. Activation of liver X receptor (LXR) inhibits receptor activator of nuclear factor $\mathrm{kB}$ ligand (RANKL)-induced osteoclast differentiation in an LXR $\beta$-dependent mechanism. J Biol Chem. 2011;286(38):33084-33094.

44. Scholtysek C, et al. PPARbeta/delta governs Wnt signaling and bone turnover. Nat Med. 2013;19(5):608-613.

45. Dawson MI, Xia Z. The retinoid X receptors and their ligands. Biochim Biophys Acta. 2012;1821(1):21-56.

46. Rahman MM, Bhattacharya A, Banu J, Kang JX, Fernandes G. Endogenous n-3 fatty acids protect ovariectomy induced bone loss by attenuating osteoclastogenesis. J Cell Mol Med. 2009;13(8):1833-1844.

47. Kelly OJ, Gilman JC, Kim Y, Ilich JZ. Long-chain polyunsaturated fatty acids may mutually benefit both obesity and osteoporosis. Nutr Res. 2013;33(7):521-533.

48. Conaway HH, et al. Retinoids inhibit differentiation of hematopoietic osteoclast progenitors. FASEB J. 2009;23(10):3526-3538.

49. Hu L, Lind T, Sundqvist A, Jacobson A, Melhus H. Retinoic acid increases proliferation of human osteoclast progenitors and inhibits RANKL-stimulated osteoclast differentiation by suppressing RANK. PLoS One. 2010;5(10):e13305.

50. Eberle D, Hegarty B, Bossard P, Ferre P, Foufelle F. SREBP transcription factors: master regulators of lipid homeostasis. Biochimie. 2004;86(11):839-848.

51. Cornish J, et al. Modulation of osteoclastogenesis by fatty acids. Endocrinology. 2008;149(11):5688-5695.

52. Bab I, Smoum R, Bradshaw H, Mechoulam R. Skeletal lipidomics: regulation of bone metabolism by fatty acid amide family. Br J Pharmacol. 2011;163(7):1441-1446.

53. Grasser WA, et al. Regulation of osteoclast dif- ferentiation by statins. J Musculoskelet Neuronal Interact. 2003;3(1):53-62.

54. Mukherjee R, et al. Sensitization of diabetic and obese mice to insulin by retinoid $\mathrm{X}$ receptor agonists. Nature. 1997;386(6623):407-410.

55. Li M, Messaddeq N, Teletin M, Pasquali JL, Metzger D, Chambon P. Retinoid X receptor ablation in adult mouse keratinocytes generates an atopic dermatitis triggered by thymic stromal lymphopoietin. Proc Natl Acad Sci U S A. 2005;102(41):14795-14800.

56. Peet DJ, et al. Cholesterol and bile acid metabolism are impaired in mice lacking the nuclear oxysterol receptor LXRa. Cell.1998;93(5):693-704.

57. Pascual-Garcia M, et al. Liver X receptors inhibit macrophage proliferation through downregulation of cyclins D1 and B1 and cyclin-dependent kinases 2 and 4. J Immunol. 2011;186(8):4656-4667.

58. Akiyama TE, et al. Conditional disruption of the peroxisome proliferator-activated receptor $\gamma$ gene in mice results in lowered expression of ABCA1, ABCG1, and apoE in macrophages and reduced cholesterol efflux. Mol Cell Biol. 2002;22(8):2607-2619.

59. Fuchs H, Lisse T, Abe K, De Angelis MH. Screening for bone and cartilage phenotypes in mice. In: De Angelis MH, Chambon P, Brown S, eds. Standards of Mouse Model Phenotyping. Datenschutz, Germany: Wiley-VCH Verlag GmbH; 2008:35-86.

60. Sontag W. Quantitative measurement of periosteal and cortical-endosteal bone formation and resorption in the midshaft of female rat femur. Bone. 1986;7(1):55-62.

61. Rőszer T, et al. Autoimmune kidney disease and impaired engulfment of apoptotic cells in mice with macrophage peroxisome proliferator-activated receptor gamma or retinoid $\mathrm{X}$ receptor $\alpha$ deficiency. JImmunol. 2011;186(1):621-631.

62. Nikolic T, de Bruijn MF, Lutz MB, Leenen PJ. Developmental stages of myeloid dendritic cells in mouse bone marrow. Int Immunol. 2003;15(4):515-524.

63. Azzout-Marniche D, Becard D, Guichard C, Foretz $\mathrm{M}$, Ferre P, Foufelle F. Insulin effects on sterol regulatory-element-binding protein-1c (SREBP1c) transcriptional activity in rat hepatocytes. Biochem J. 2000;350(pt 2):389-393.

64. Ovcharenko I, et al. Mulan: multiple-sequence local alignment and visualization for studying function and evolution. Genome Res. 2005;15(1):184-194.

65 . Heinz S, et al. Simple combinations of lineagedetermining transcription factors prime cis-regulatory elements required for macrophage and $\mathrm{B}$ cell identities. Mol Cell. 2010;38(4):576-589.

66. Ricote M, et al. Expression of the peroxisome proliferator-activated receptor g (PPARg) in human atherosclerosis and regulation in macrophages by colony stimulating factors and oxidized low density lipoprotein. Proc Natl Acad Sci U S A. 1998;95(13):7614-7619.

67. Lefterova MI, et al. Cell-specific determinants of peroxisome proliferator-activated receptor gamma function in adipocytes and macrophages. Mol Cell Biol. 2010;30(9):2078-2089.

68. Fuller K, Kirstein B, Chambers TJ. Murine osteoclast formation and function: differential regulation by humoral agents. Endocrinology. 2006;147(4):1979-1985. 